Geometry $\&$ Topology

Volume 9 (2005) 2159-2191

Published: 22 November 2005

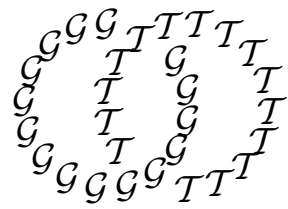

\title{
Homology and derived series of groups
}

\author{
TiM COCHRAN \\ SHELly HARVEY \\ Department of Mathematics, Rice University \\ Houston, TX 77005-1892, USA
}

Email: cochran@math.rice.edu and shelly@math.rice.edu

\begin{abstract}
In 1964, John Stallings established an important relationship between the lowdimensional homology of a group and its lower central series. We establish a similar relationship between the low-dimensional homology of a group and its derived series. We also define a torsion-free-solvable completion of a group that is analogous to the Malcev completion, with the role of the lower central series replaced by the derived series. We prove that the torsion-free-solvable completion is invariant under rational homology equivalence.
\end{abstract}

AMS Classification numbers Primary: 20J06

Secondary: $57 \mathrm{M} 07,55 \mathrm{P} 60$

Keywords: Derived series, group homology, Malcev completion, homological localization

Proposed: Robion Kirby

Seconded: Cameron Gordon, Joan Birman
Received: 27 January 2005

Revised: 11 November 2005 


\section{Introduction}

John Stallings, in his landmark paper [25], established the following relationships between the low-dimensional homology of a group and its lower central series. Recall that, for any ordinal $\alpha$, the $\alpha^{\text {th }}$ term of the lower central series of $G$, denoted $G_{\alpha}$, is inductively defined by $G_{1}=G, G_{\alpha+1}=\left[G_{\alpha}, G\right]$ and, if $\alpha$ is a limit ordinal, $G_{\alpha}=\bigcap_{\beta<\alpha} G_{\beta}$. Stallings also defines what we shall call the rational lower central series, $G_{\alpha}^{r}$, which differs only in that $G_{\alpha+1}^{r}$ consists of all those elements some finite power of which lies in $\left[G_{\alpha}^{r}, G\right]$. It is the most rapidly descending central series whose successive quotients are torsion free abelian groups.

Stallings' Theorem (Integral) [25, Theorem 3.4] Let $\phi: A \rightarrow B$ be a homomorphism that induces an isomorphism on $H_{1}(-; \mathbb{Z})$ and an epimorphism on $H_{2}(-; \mathbb{Z})$. Then, for any finite $n, \phi$ induces an isomorphism $A / A_{n} \cong B / B_{n}$. For the first infinite ordinal $\omega$, it induces an embedding $A / A_{\omega} \subset B / B_{\omega}$. If, in addition, $\phi$ is onto then, for each ordinal $\alpha, \phi$ induces an isomorphism $A / A_{\alpha} \cong B / B_{\alpha}$.

Stallings' Theorem (Rational) [25. Theorem 7.3] Let $\phi: A \rightarrow B$ be a homomorphism that induces an isomorphism on $H_{1}(-; \mathbb{Q})$ and an epimorphism on $H_{2}(-; \mathbb{Q})$. Then, for all $\alpha \leq \omega, \phi$ induces an embedding $A / A_{\alpha}^{r} \subset B / B_{\alpha}^{r}$ and for any finite $n$ it induces isomorphisms $\left(A_{n}^{r} / A_{n+1}^{r}\right) \otimes \mathbb{Q} \cong\left(B_{n}^{r} / B_{n+1}^{r}\right) \otimes \mathbb{Q}$.

These theorems have proven to be quite useful in topology. For example if $A=\pi_{1}\left(S^{3} \backslash L\right)$ where $L$ is a link of circles in $S^{3}$, Stallings showed that the isomorphism type of each of the quotients $A / A_{n}$ is an invariant of link concordance (even of $I$-equivalence). The concordance invariance of Milnor's $\bar{\mu}$-invariants was established by this means 3 . Stallings' theorems also give a criterion for establishing that a collection of elements of a group generates a free subgroup.

Attempts have been made, most notably by Ralph Strebel [27, to find a similar relationship between homology and the derived series of groups, with limited success. We will use the work of Strebel in a crucial way. Recall that the $\alpha^{\text {th }}$ term of the derived series, $G^{(\alpha)}$, is defined by $G^{(0)}=G, G^{(\alpha+1)}=\left[G^{(\alpha)}, G^{(\alpha)}\right]$ and, if $\alpha$ is a limit ordinal, $G^{(\alpha)}=\bigcap_{\beta<\alpha} G^{(\beta)}$. The derived series has recently appeared prominently in joint work of the first author, Kent Orr and Peter Teichner [6] [7, 8] 28] where it was used to define new invariants for classical knot concordance, and in work by other authors 15 16. It was was also used to define higher-order Alexander invariants for knots [5], and 3-manifolds [12, 
and to define invariants for link concordance and rational homology cobordism of manifolds [11. It has also appeared recently in connection with questions about the virtual first Betti number of 3-manifolds [24] and in the study of complements of hyperplane arrangements [21].

We show that there is a strong relationship between the low-dimensional homology of a group and its derived series. For example, we have the following strict analogue of Stallings' Rational Theorem in the case $A$ is a free group.

Corollary 4.5 Let $F$ be a free group and $B$ be a finitely related group (has a presentation with a finite number of relations). Let $\phi: F \rightarrow B$ be a homomorphism that induces a monomorphism on $H_{1}(-; \mathbb{Q})$ and an epimorphism on $H_{2}(-; \mathbb{Q})$. Then, for all $\alpha \leq \omega, \phi$ induces an embedding $F / F^{(\alpha)} \subset B / B^{(\alpha)}$.

The following examples show that several obvious generalizations of Corollary 4.5] are false. Let $K$ be any knot in $S^{3}, A=\pi_{1}\left(S^{3} \backslash K\right)$ and $B=\mathbb{Z}$. Then the abelianization map yields a homomorphism $\phi: A \rightarrow A /[A, A] \cong \mathbb{Z}=B$. Then $\phi$ induces an isomorphism on all integral homology groups (since, by Alexander Duality, $S^{3} \backslash K$ has the homology of a circle) and $B^{(n)}=\{e\}$ for any $n \geq 1$; whereas $A / A^{(n)}$ is known to be very large as long as the Alexander polynomial of $K$ is not 1 [5. Corollary 4.8]. Thus $\phi$ cannot induce monomorphisms in general as in Corollary 4.5. Moreover, swapping the roles of $A$ and $B$ and choosing a map $\mathbb{Z} \rightarrow A$ inducing an isomorphism on $H_{1}(-; \mathbb{Z})$ gives a homomorphism that again induces isomorphisms on all integral homology groups and yet induces the map $\mathbb{Z} \rightarrow A / A^{(n)}$ for each $n$, this being far from surjective. Thus a direct analogue of Stallings' theorem might seem hopeless.

However the second author, in a search for new invariants of link concordance, introduced a new characteristic series, $G_{H}^{(n)}$, associated to the derived series, called the torsion-free derived series (see [11, Section 2]). Although this series is not fully invariant, we show that it is functorial when morphisms are restricted to those that induce a monomorphism on $H_{1}(-; \mathbb{Q})$ and an epimorphism on $H_{1}(-; \mathbb{Q})$. Using this we are able show the following analogue of Stallings' Rational Theorem.

Theorem 4.1 Let $A$ be finitely-generated and $B$ finitely related (has a presentation with a finite number of relations). Let $\phi: A \rightarrow B$ be a homomorphism that induces a monomorphism on $H_{1}(-; \mathbb{Q})$ and an epimorphism on $H_{2}(-; \mathbb{Q})$. Then, for each $n \leq \omega, \phi$ induces a monomorphism $A / A_{H}^{(n)} \subset B / B_{H}^{(n)}$. Moreover, if $\phi$ induces an isomorphism on $H_{1}(-; \mathbb{Q})$ then, for each finite $n$, $A_{H}^{(n)} / A_{H}^{(n+1)} \rightarrow B_{H}^{(n)} / B_{H}^{(n+1)}$ is a monomorphism between modules of the same 
rank (over $\mathbb{Z}\left[A / A_{H}^{(n)}\right]$ and $\mathbb{Z}\left[B / B_{H}^{(n)}\right]$, respectively). If, in addition $\phi$ is onto, then for each $n \leq \omega$, it induces an isomorphism $A / A_{H}^{(n)} \cong B / B_{H}^{(n)}$.

Corollary 4.5 is a special case of Theorem 4.1.

Note that, by Stallings' Integral Theorem, the (pro-)nilpotent completion of a group $G, \lim \left(G / G_{n}\right)$ is an invariant of integral homology equivalence. But neither the nilpotent completion nor its torsion-free version $\lim \left(G / G_{n}^{r}\right)$ is invariant under rational homology equivalence (for example the rational homology equivalence $\mathbb{Z} \rightarrow \mathbb{Z}$ where $t \rightarrow t^{2}$ does not induce an isomorphism on these completions). However there does exist a further completion, the Malcev completion of $G$, which is invariant under rational homology equivalence (a consequence of the last part of Stallings' Rational Theorem). Turning to the derived series, the examples above show that neither the pro-solvable completion, $\lim \left(G / G^{(n)}\right)$, nor its torsion-free version, $\underset{\lim }{\longleftarrow}\left(G / G_{r}^{(n)}\right)$, nor even our $\lim _{(}\left(G / G_{H}^{(n)}\right)$ (see Section 2) is invariant under rational (or even integral) homology equivalence. However, early versions of [1] suggested another functor on groups, $G \rightarrow \widetilde{G}$, that we call the torsion-free-solvable completion of $G$. This is properly viewed as an analogue of the Malcev completion of $G$, with the lower central series being replaced by the torsion-free derived series $G_{H}^{(n)}$. Here we rigorously construct $G \rightarrow \widetilde{G}$, whose kernel is $G_{H}^{(\omega)}$, and show that it is a rational homological localization functor, that is we show:

Theorem 5.1] Suppose $A$ is finitely generated and $B$ is finitely related. Suppose $\phi: A \rightarrow B$ induces an isomorphism on $H_{1}(-; \mathbb{Q})$ and an epimorphism on $H_{2}(-; \mathbb{Q})$. Then $\phi$ induces an isomorphism $\widetilde{A} \cong \widetilde{B}$.

We also give the precise relationship between the torsion-free-solvable completion and the universal (integral) homological localization functor due to J Levine and P Vogel, and indicate the relation (by analogy) between the torsionfree-solvable completion and the Malcev completion.

Acknowledgements The first author was partially supported by NSF DMS0406573. The second author was partially supported by an NSF Postdoctoral Fellowship and NSF DMS-0539044.

\section{The torsion-free derived series}

In this section we motivate and define Harvey's version of the derived series, $G_{H}^{(n)}$, and remark on some of its elementary properties. 
If $G$ is a group then $G / G^{(1)}$ is an abelian group but may have $\mathbb{Z}$-torsion. If one would like to avoid this $\mathbb{Z}$-torsion then, in direct analogy to the rational lower-central series above, one can define $G_{r}^{(1)}=\left\{x \mid \exists k \neq 0 x^{k} \in G^{(1)}\right\}$, slightly larger than $G^{(1)}$, so that $G / G_{r}^{(1)}$ is $\mathbb{Z}$-torsion-free. Proceeding in this way, defining $G_{r}^{(n+1)}$ to be the radical of $\left[G_{r}^{(n)}, G_{r}^{(n)}\right]$, leads to what has been called the rational derived series of $G$ [12, [5, 8]. This is the most rapidly descending series for which the quotients of successive terms are $\mathbb{Z}$-torsion-free abelian groups. In [1] Harvey observes that if a subgroup $G_{H}^{(n)}$ (normal in $G$ ) has been defined then $G_{H}^{(n)} /\left[G_{H}^{(n)}, G_{H}^{(n)}\right]$ is not only an abelian group but also a right module over $\mathbb{Z}\left[G / G_{H}^{(n)}\right]$, where the action is induced from the conjugation action of $G\left([x] g=\left[g^{-1} x g\right]\right)$. One might seek to eliminate torsion in the module sense from the successive quotients. This motivated her definition of the torsion-free derived series as follows. Set $G_{H}^{(0)}=G$. For $n \geq 0$, suppose inductively that $G_{H}^{(n)}$ has been defined and is normal in $G$. Let $T_{n}$ be the subset of $G_{H}^{(n)} /\left[G_{H}^{(n)}, G_{H}^{(n)}\right]$ consisting of the $\mathbb{Z}\left[G / G_{H}^{(n)}\right]$-torsion elements, ie, the elements $[x]$ for which there exists some non-zero $\gamma \in \mathbb{Z}\left[G / G_{H}^{(n)}\right]$, such that $[x] \gamma=0$. (In fact, since it will be (inductively) shown below that $\mathbb{Z}\left[G / G_{H}^{(n)}\right]$ is an Ore Domain, $T_{n}$ is a submodule). Now consider the epimorphism of groups:

$$
G_{H}^{(n)} \stackrel{\pi_{n}}{\longrightarrow} \frac{G_{H}^{(n)}}{\left[G_{H}^{(n)}, G_{H}^{(n)}\right]}
$$

and define $G_{H}^{(n+1)}$ to be the inverse image of $T_{n}$ under $\pi_{n}$. Then $G_{H}^{(n+1)}$ is, by definition, a normal subgroup of $G_{H}^{(n)}$ that contains $\left[G_{H}^{(n)}, G_{H}^{(n)}\right]$. It follows inductively that $G_{H}^{(n+1)}$ contains $G^{(n+1)}\left(\right.$ and $\left.G_{r}^{(n+1)}\right)$. Moreover, since $G_{H}^{(n)} / G_{H}^{(n+1)}$ is the quotient of the module $G_{H}^{(n)} /\left[G_{H}^{(n)}, G_{H}^{(n)}\right]$ by its torsion submodule, it is a $\mathbb{Z}\left[G / G_{H}^{(n)}\right]$ torsion-free module [26, Lemma 3.4]. Hence the successive quotients of the torsion-free derived subgroups are torsion-free modules over the appropriate rings. We define $G_{H}^{(\omega)}=\bigcap_{n<\omega} G_{H}^{(n)}$ as usual. However a discussion of $G_{H}^{(\alpha)}$ for $\alpha>\omega$ would require further work since $\mathbb{Z}\left[G / G_{H}^{(\omega)}\right]$ is not in general an Ore Domain. We do not address this here.

Most of the following elementary properties of the torsion-free derived series were established in 11. We repeat the proofs for the convenience of the reader.

Proposition 2.1 11] $G / G_{H}^{(n)}$ is a poly-(torsion-free abelian) group (hereafter abbreviated PTFA), and consequently $\mathbb{Z}\left[G / G_{H}^{(n)}\right]$ is an Ore domain.

Recall that a poly-(torsion-free abelian) group is one with a finite subnormal series whose successive quotients are torsion-free abelian groups. Such a group is solvable, torsion free and locally indicable [27, Proposition 1.9]. Consequently 
$\mathbb{Z}\left[G / G_{H}^{(n)}\right]$ is an Ore domain and thus admits a classical (right) division ring of fractions, $\mathcal{K}\left(G / G_{H}^{(n)}\right)$, into which it embeds [22, pages 591-592] [22, page 611] 19]. Hence any (right) module $M$ over $\mathbb{Z}\left[G / G_{H}^{(n)}\right]$ has a well-defined rank which is defined to be the rank of the vector space $M \otimes_{\mathbb{Z}\left[G / G_{H}^{(n)}\right]} \mathcal{K}\left(G / G_{H}^{(n)}\right)$, 9 , page 48]. Alternatively the rank can be defined to be the maximal integer $m$ such that $M$ contains a submodule isomorphic to $\left(\mathbb{Z}\left[G / G_{H}^{(n)}\right]\right)^{m}$.

Proposition 2.2 11] $G_{H}^{(n+1)}$ is a normal subgroup of $G$.

Proof of Proposition 2.2 The proof is by induction on $n$. We assume $G_{H}^{(n)}$ is normal in $G$ and show that $G_{H}^{(n+1)}$ is normal in $G$. Consider $x \in G_{H}^{(n+1)}$ and $g \in G$. Then $x$ and $g^{-1} x g$ lie in $G_{H}^{(n)}$ and, by definition of the module structure on $G_{H}^{(n)} /\left[G_{H}^{(n)}, G_{H}^{(n)}\right], \pi_{n}\left(g^{-1} x g\right)=\pi_{n}(x) g$. Since $x \in G_{H}^{(n+1)}, \pi_{n}(x)$ is torsion. To show that $g^{-1} x g \in G_{H}^{(n+1)}$, we need to show that $\pi_{n}(x) g$ is torsion. Hence the normality of $G_{H}^{(n+1)}$ will follow from showing that the torsion subgroup $T_{n}$ is a submodule. But the set of torsion elements of any module over an Ore domain is known to be a submodule [26, page 57]. Thus the desired result follows from Proposition 2.1

The torsion-free derived subgroups are characteristic subgroups, but are not fully invariant, that is an arbitrary homomorphism of groups $\phi: A \longrightarrow B$ need not send $A_{H}^{(n)}$ into $B_{H}^{(n)}$. The simplest example is $A=\langle x, y, z \mid[z,[x, y]]=1\rangle$, $B=\langle x, y\rangle$, with $\phi$ sending $(x \rightarrow x, y \rightarrow y, z \rightarrow 0)$. Then $[x, y] \in A_{H}^{(2)}$ but $\phi([x, y])$ is not in $B_{H}^{(2)}=B^{(2)}$. However, the following observation is used in our main theorem to show (inductively) that if $\phi: A \rightarrow B$ induces a monomorphism on $H_{1}(-; \mathbb{Q})$ and an epimorphism on $H_{2}(-; \mathbb{Q})$, and if $A$ is finitely generated and $B$ finitely related, then $\phi$ does send $A_{H}^{(n)}$ into $B_{H}^{(n)}$. It follows that the torsion-free derived series is functorial in this restricted category.

Proposition 2.3 [1] If $\phi: A \rightarrow B$ induces a monomorphism $\phi: A / A_{H}^{(n)} \rightarrow$ $B / B_{H}^{(n)}$, then $\phi\left(A_{H}^{(n+1)}\right) \subset B_{H}^{(n+1)}$ and hence $\phi$ induces a homomorphism $\phi$ : $A / A_{H}^{(n+1)} \rightarrow B / B_{H}^{(n+1)}$.

Proof of Proposition 2.3 Note that the hypothesis implies that $\phi$ induces a ring monomorphism $\tilde{\phi}: \mathbb{Z}\left[A / A_{H}^{(n)}\right] \rightarrow \mathbb{Z}\left[B / B_{H}^{(n)}\right]$. Suppose that $x \in A_{H}^{(n+1)}$. Consider the diagram below.

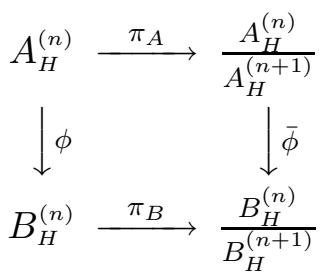


By definition, $\pi_{A}(x)$ is torsion, that is there is some non-zero $\gamma \in \mathbb{Z}\left[A / A_{H}^{(n)}\right]$ such that $\left(\pi_{A}(x)\right) \gamma=0$. It is easy to check that $\bar{\phi}$ is a homomorphism of right $\mathbb{Z}\left[A / A_{H}^{(n)}\right]$-modules using the module structure induced on $B_{H}^{(n)} / B_{H}^{(n+1)}$ by $\tilde{\phi}$ (since $\left.\phi\left(a^{-1} x a\right)=\phi(a)^{-1} \phi(x) \phi(a)\right)$. Thus $\left(\bar{\phi}\left(\pi_{A}(x)\right)\right) \tilde{\phi}(\gamma)=0$ and so, since $\tilde{\phi}$ is injective, $\bar{\phi}\left(\pi_{A}(x)\right)$ is a $\mathbb{Z}\left[B / B_{H}^{(n)}\right]$-torsion element. But $\bar{\phi}\left(\pi_{A}(x)\right)=$ $\pi_{B}(\phi(x))$, showing that $\phi(x) \in B_{H}^{(n+1)}$. Hence $\phi\left(A_{H}^{(n+1)}\right) \subset B_{H}^{(n+1)}$.

For some groups, such as free groups and free-solvable groups $F / F^{(k)}$, the derived series and the torsion-free derived series coincide.

Proposition 2.4 [1] If $G$ is a group such that, for every $n, G^{(n)} / G^{(n+1)}$ is a $\mathbb{Z}\left[G / G^{(n)}\right]$-torsion-free module, then the torsion-free derived series of $G$ agrees with the derived series of $G$. Hence for a free group $F, F_{H}^{(n)}=F^{(n)}$ for each $n$, and similarly for a free solvable group $F / F^{(k)}$.

Proof of Proposition 2.4 By definition, $G_{H}^{(0)}=G^{(0)}=G$. Suppose $G_{H}^{(n)}=$ $G^{(n)}$. Then, under the hypotheses, $G_{H}^{(n)} /\left[G_{H}^{(n)}, G_{H}^{(n)}\right]$ is a torsion-free module and so $G_{H}^{(n+1)}=\operatorname{ker} \pi_{n}=\left[G_{H}^{(n)}, G_{H}^{(n)}\right]=\left[G^{(n)}, G^{(n)}\right]=G^{(n+1)}$.

It is well known that $F^{(n)} / F^{(n+1)}$ is a $\mathbb{Z}\left[F / F^{(n)}\right]$-torsion-free module. This can be seen by examining the free $\mathbb{Z}\left[F / F^{(n)}\right]$ cellular chain complex for the covering space of a wedge of circles corresponding to the subgroup $F^{(n)}$. The module $F^{(n)} / F^{(n+1)}$ is merely the first homology of this chain complex. Since the chain complex can be chosen to have no 2-cells, its first homology is a submodule of a free module and thus is torsion-free. Hence the derived series and the torsionfree derived series of a free group agree. The same is true for any free-solvable group $F / F^{(k)}$.

The following basic result is useful.

Proposition 2.5 If $\phi: A \rightarrow B$ is surjective and $\operatorname{ker} \phi \subset A_{H}^{(m)}$ then $\phi$ induces isomorphisms $A / A_{H}^{(n)} \cong B / B_{H}^{(n)}$ for all $n \leq m$. In particular, $\left(G / G_{H}^{(n)}\right)_{H}^{(n)}=0$.

Proof of Proposition 2.5 By induction we assume $\phi$ induces isomorphisms $A / A_{H}^{(i)} \cong B / B_{H}^{(i)}$ for each $i \leq n$ for some $0 \leq n<m$. Since $\phi$ induces a $\operatorname{map} A / A_{H}^{(n)} \rightarrow B / B_{H}^{(n)}$ surely $\phi\left(A_{H}^{(n)}\right) \subset B_{H}^{(n)}$. We claim that $\phi\left(A_{H}^{(n)}\right)=B_{H}^{(n)}$, because for any $b \in B_{H}^{(n)}$ there is an $a \in A$ such that $\phi(a)=b$ and, by the inductive hypothesis, we must have $a \in A_{H}^{(n)}$. Therefore it also follows that $\phi\left(\left[A_{H}^{(n)}, A_{H}^{(n)}\right]\right)=\left[B_{H}^{(n)}, B_{H}^{(n)}\right]$, a fact we use below. Moreover, by Proposition 2.3 , $\phi\left(A_{H}^{(n+1)}\right) \subset B_{H}^{(n+1)}$ and so induces an epimorphism $\phi: A / A_{H}^{(n+1)} \rightarrow B / B_{H}^{(n+1)}$. 
We need to show this is injective to complete the proof. Suppose there exists $a \in A$ such that $\phi(a)=b \in B_{H}^{(n+1)}$. By the inductive hypothesis, $a \in A_{H}^{(n)}$. Refer to the commutative diagram below.

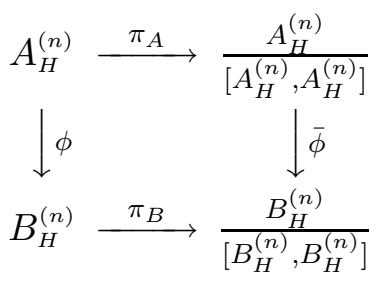

Since $b \in B_{H}^{(n+1)}, \pi_{B}(b)$ is a torsion element and so there exists a non-zero $\gamma \in \mathbb{Z}\left[B / B_{H}^{(n)}\right]$ such that $\left(\pi_{B}(b)\right) \gamma=0$. We seek to express this entirely in terms of $B$ itself. Lift $\gamma$ to $\mathbb{Z} B$ and express it as $\sum k_{i} \eta_{i}$ where $k_{i} \in \mathbb{Z}$ and $\eta_{i} \in B$. The condition that $\left(\pi_{B}(b)\right) \gamma=0$ translates to the condition $\prod\left(\eta_{i}^{-1} b^{k_{i}} \eta_{i}\right)=z$ for some $z \in\left[B_{H}^{(n)}, B_{H}^{(n)}\right]$. Since $\phi$ is surjective, $\eta_{i}=\phi\left(\alpha_{i}\right)$ for some $\alpha_{i} \in A$ and $z=\phi(x)$ for some $x \in\left[A_{H}^{(n)}, A_{H}^{(n)}\right]$ by our remark above. Then $x^{-1} \prod\left(\alpha_{i}^{-1} a^{k_{i}} \alpha_{i}\right)$ is in the kernel of $\phi$ and hence by hypothesis lies in $A_{H}^{(m)} \subset A_{H}^{(n+1)}$. Since $x \in\left[A_{H}^{(n)}, A_{H}^{(n)}\right] \subset A_{H}^{(n+1)}$, it follows that $\prod\left(\alpha_{i}^{-1} a^{k_{i}} \alpha_{i}\right)$ is in $A_{H}^{(n+1)}$. Let $\beta=$ $\sum k_{i} \alpha_{i} \in \mathbb{Z}\left[A / A_{H}^{(n)}\right]$ which is non-zero since $\phi(\beta)=\gamma$ is non-zero. Note that $\pi_{A}(a) \beta$ is represented by $\prod\left(\alpha_{i}^{-1} a^{k_{i}} \alpha_{i}\right)$. Thus we have shown that $\pi_{A}(a) \beta$ is zero when mapped into $A_{H}^{(n)} / A_{H}^{(n+1)}$ and hence that the image of $\pi_{A}(a)$ is torsion in this module. But the successive quotients of terms in the torsion-free derived series are torsion-free modules as remarked earlier, so $\pi_{A}(a)=0$ in this module. Therefore $a \in A_{H}^{(n+1)}$, completing the proof of injectivity.

To prove that $\left(G / G_{H}^{(n)}\right)_{H}^{(n)}=0$, apply the above to the $\phi: G \rightarrow G / G_{H}^{(n)}$.

We give some elementary examples of the torsion-free derived series.

Example 2.6 Elements of finite order in a group $G$ are contained in every term of the rational derived series and hence every term of the torsion-free derived series. Therefore the torsion-free derived series of a finite group stabilizes at $n=0$, that is $G_{H}^{(n)}=G_{H}^{(0)}=G$. In general $G / G_{H}^{(\omega)}$ is a torsion-free group that is trivial if $G$ is finite. More generally, if $\beta_{1}(G)=0$, that is if $H_{1}(G ; \mathbb{Q})=0$, then the torsion-free derived series, like the rational derived series, stabilizes at $n=0$.

Example 2.7 If $G$ is nilpotent with $\beta_{1}(G)>0$ then the terms of the torsionfree derived series are much larger than the terms of the rational derived series. In this case the torsion-free derived series stabilizes at $n=1$. For suppose $x \in G$ is an arbitrary element of $G_{H}^{(n)}$ for some $n \geq 1$ and $t \in G$ is non-zero 
in $G / G_{H}^{(1)}$. We will show that $x \in G_{H}^{(n+1)}$. Since $\mathrm{G}$ is nilpotent, for some $k$ the simple $(k+1)$-commutator, $[t,[t,[\ldots[t, x] \ldots]]]$ is the identity in $G$. In the module $G_{H}^{(n)} /\left[G_{H}^{(n)}, G_{H}^{(n)}\right]$ this gives the relation $(t-1)^{k} x=0$, showing that $x$ represents a torsion element (since $(t-1)^{k}$ is non-zero in the integral domain $\left.\mathbb{Z}\left[G / G_{H}^{(n)}\right]\right)$. Hence $x \in G_{H}^{(n+1)}$.

Example 2.8 If $G$ is free-solvable of class $n$, that is $G=F / F^{(n)}$ where $F$ is a non-abelian free group, then by Proposition 2.4 and Proposition 2.5 . $G / G_{H}^{(m)} \cong F / F^{(m)}$ for all $m \leq n$ and $G^{(n)}=0$. Thus the torsion-free derived series stabilizes at precisely $n$.

Example 2.9 If $\beta_{1}(G)=1$, for example if $G$ is the fundamental group of the exterior of a knotted circle in $S^{3}$, then the torsion-free derived series stabilizes at $n=1$ because the classical Alexander module, $G_{H}^{(1)} /\left[G_{H}^{(1)}, G_{H}^{(1)}\right]$ can be seen to be a torsion module over $\mathbb{Z}\left[t, t^{-1}\right]$ (see, for example, 6 , Proposition 2.1]). Thus $G_{H}^{(2)}=G_{H}^{(1)}$. Alternatively, note that the epimorphism $G \rightarrow \mathbb{Z}$ induces an isomorphism on $H_{1}(-; \mathbb{Q})$ and an epimorphism on $H_{2}(-; \mathbb{Q})$ and so, by Theorem 4.1, it induces isomorphisms $G / G_{H}^{(n)} \cong \mathbb{Z}$.

Example 2.10 Generalizing Example 2.9. if $G$ is any group whose classical Alexander module $G_{H}^{(1)} /\left[G_{H}^{(1)}, G_{H}^{(1)}\right]$ has rank zero (over the multivariable Laurent polynomial ring $\left.\mathbb{Z}\left[t_{1}^{ \pm 1}, \ldots, t_{m}^{ \pm 1}\right]\right)$ then the torsion-free derived series stabilizes at $n \leq 1$. The fundamental group of any 3 -manifold that fibers over a circle has this property [12, Proposition 8.4]. By contrast, the derived series of the fundamental group of a knot exterior does not stabilize for any finite $n$ unless the Alexander polynomial is 1 [5, Corollary 4.8]. More generally, if the rank of one of the higher-order modules $G_{H}^{(n)} /\left[G_{H}^{(n)}, G_{H}^{(n)}\right]$, is zero then the torsion-free derived series stabilizes at the minimum value of $n$ for which this holds.

Example 2.11 For groups with $\beta_{1}(G) \geq 2$, a wide variety of behavior is possible. For example, as previously mentioned, the torsion-free derived series of a non-abelian free group, $F$, agrees with the derived series and is known to be highly non-trivial, stabilizing at $\omega$ (in fact $F_{H}^{(\omega)}=0$ ). There are many nonfree groups where the torsion-free derived series does not stabilize at a finite ordinal. Recall that a link $\left\{L_{i}\right\}$ of $m$ circles in $S^{3}$ is called a boundary link if there is a collection of $m$ compact oriented surfaces $\left\{V_{i}\right\}$, disjointly embedded in $S^{3}$ such that $\partial V_{i}=L_{i}$. If $G=\pi_{1}\left(S^{3}-L\right)$ then the meridional map $F \rightarrow G$ has a right inverse $G \rightarrow F$ (use the Pontryagin construction to get a map from $S^{3}-L$ to a wedge of circles). The latter epimorphism induces an isomorphism 
on $H_{1}(-; \mathbb{Z})$ and an epimorphism $H_{2}(-; \mathbb{Z})$ and so, by Theorem 4.1, for $n \leq \omega$, $G / G_{H}^{(n)} \cong F / F^{(n)}$ and $G / G_{H}^{(\omega)} \cong F$. It follows from Stallings' Integral Theorem that $G_{H}^{(\omega)}=G_{\omega}$. Since for a boundary link it is known that $G_{\omega} /\left[G_{\omega}, G_{\omega}\right]$ is a torsion module, the torsion-free derived series stabilizes at $\omega$. However, suppose $G$ is the fundamental group of a 2 -complex $X$ with $H_{1}(X ; \mathbb{Z}) \cong \mathbb{Z}^{m}$ and $H_{2}(X ; \mathbb{Z})=0$. Then there is a map $F \rightarrow G$ inducing isomorphisms on $H_{1}(-; \mathbb{Z})$ and $H_{2}(-; \mathbb{Z})$. Thus, by Theorem 4.1 $F / F^{(n)} \subset G / G_{H}^{(n)}$. However generally this is not surjective and $G_{H}^{(n)} / G_{H}^{(n+1)}$ is generally not even abstractly isomorphic to $F^{(n)} / F^{(n+1)}$ (see Remark 4.6). In these cases it is not in general known if the torsion-free derived series stabilizes at $\omega$.

It will be important for our main theorem that the reader understand the connection between the torsion-free derived series and group homology. This is provided by the following basic observations.

Remark 2.12 $A_{H}^{(n)} /\left[A_{H}^{(n)}, A_{H}^{(n)}\right] \cong H_{1}\left(A ; \mathbb{Z}\left[A / A_{H}^{(n)}\right]\right)$. For an algebraist this is a consequence of the definition $H_{1}\left(A ; \mathbb{Z}\left[A / A_{H}^{(n)}\right]\right) \equiv \operatorname{Tor}_{1}^{A}\left(\mathbb{Z}\left[A / A_{H}^{(n)}\right], \mathbb{Z}\right)$ and the easy observation that the latter is $\operatorname{Tor}_{1}^{A_{H}^{(n)}}(\mathbb{Z}, \mathbb{Z}) \cong A_{H}^{(n)} /\left[A_{H}^{(n)}, A_{H}^{(n)}\right][13$, Lemma $6.2]$. For a topologist, $H_{1}\left(A ; \mathbb{Z}\left[A / A_{H}^{(n)}\right]\right)$ is thought of as the first homology with twisted coefficients of an aspherical space $K(A, 1)$ where $\pi_{1}(K(A, 1)) \cong A$ and the coefficient system is induced by $\pi_{1}(K(A, 1)) \cong A \rightarrow A / A_{H}^{(n)}$ [13, page 335]. Then $H_{1}\left(K(A, 1) ; \mathbb{Z}\left[A / A_{H}^{(n)}\right]\right)$ can be interpreted as the first homology module of the covering space of $\mathrm{K}(\mathrm{A}, 1)$ corresponding to the subgroup $A_{H}^{(n)}$, which is $A_{H}^{(n)} /\left[A_{H}^{(n)}, A_{H}^{(n)}\right]$ [29, Theorems VI3.4 and 3.4*].

\section{Proposition 2.13}

(1) $A_{H}^{(n)} / A_{H}^{(n+1)}$ is equal to $H_{1}\left(A ; \mathbb{Z}\left[A / A_{H}^{(n)}\right]\right)$ modulo its $\mathbb{Z}\left[A / A_{H}^{(n)}\right]$-torsion submodule.

(2) $A_{H}^{(n+1)}$ is the kernel of the composition:

$$
\begin{aligned}
A_{H}^{(n)} \stackrel{\pi_{n}}{\longrightarrow} \frac{A_{H}^{(n)}}{\left[A_{H}^{(n)}, A_{H}^{(n)}\right]} & =H_{1}\left(A ; \mathbb{Z}\left[A / A_{H}^{(n)}\right]\right) \\
& \rightarrow H_{1}\left(A ; \mathbb{Z}\left[A / A_{H}^{(n)}\right]\right) \otimes_{\mathbb{Z}\left[A / A_{H}^{(n)}\right]} \mathcal{K}\left(A / A_{H}^{(n)}\right) .
\end{aligned}
$$

Proof Property 1) follows directly from Remark 2.12 and the definition of $A_{H}^{(n+1)}$. For Property 2$)$, note that tensoring with the quotient field $\mathcal{K}\left(A / A_{H}^{(n)}\right)$ kills precisely the $\mathbb{Z}\left[A / A_{H}^{(n)}\right]$-torsion submodule [26, Corollary II.3.3]. 


\section{Topological applications}

Our theorems, like those of Stallings, have applications to the study of rational homology equivalences of topological spaces and to the study of links in particular. We also give an application to when a set of elements of a group generates a free subgroup (similar to that of Stallings). Deeper secondary applications will appear in 11.

Theorem 3.1 Let $f: X \rightarrow Y$ be a continuous map between connected $C W$ complexes that induces a monomorphism on $H_{1}(-; \mathbb{Q})$ and an epimorphism on $H_{2}(-; \mathbb{Q})$. Let $A, B$ denote $\pi_{1}(X), \pi_{1}(Y)$ respectively. Suppose that $A$ is finitely-generated and $B$ is finitely related. Then, for each $n \leq \omega, f$ induces a monomorphism $A / A_{H}^{(n)} \subset B / B_{H}^{(n)}$. If, additionally, $f$ induces an isomorphism on $H_{1}(-; \mathbb{Q})$ then, for any finite $n, A_{H}^{(n)} / A_{H}^{(n+1)} \rightarrow B_{H}^{(n)} / B_{H}^{(n+1)}$ is a monomorphism between modules of the same rank (over $\mathbb{Z}\left[A / A_{H}^{(n)}\right]$ and $\mathbb{Z}\left[B / B_{H}^{(n)}\right]$, respectively). If, in addition $f_{*}: \pi_{1}(X) \rightarrow \pi_{1}(Y)$ is onto, then, for each finite $n, f$ induces an isomorphism $A / A_{H}^{(n)} \cong B / B_{H}^{(n)}$.

Proof The theorem follows immediately from Theorem 4.1 once we make the well-known observation that since $f$ induces an epimorphism $H_{2}(X ; \mathbb{Q}) \rightarrow$ $H_{2}(Y ; \mathbb{Q})$, it induces an epimorphism $H_{2}(A ; \mathbb{Q}) \rightarrow H_{2}(B ; \mathbb{Q})$.

Corollary 3.2 If $X$ and $Y$ are $n$-manifolds (possibly with boundary) that are rationally homology cobordant (relative their boundary) via the $(n+1)$ manifold $Z$, and $A, B$ and $C$ denote their respective fundamental groups, then for any $n$, the inclusion maps induce monomorphisms $A / A_{H}^{(n)} \subset C / C_{H}^{(n)}$, and $B / B_{H}^{(n)} \subset C / C_{H}^{(n)}$. Moreover $A_{H}^{(n)} / A_{H}^{(n+1)}$ and $B_{H}^{(n)} / B_{H}^{(n+1)}$ are modules of the same rank (over $\mathbb{Z}\left[A / A_{H}^{(n)}\right]$ and $\mathbb{Z}\left[B / B_{H}^{(n)}\right]$, respectively).

In [11, Harvey uses Corollary 3.2 to give other new invariants of homology cobordism of $(2 k-1)$-manifolds, using the von Neumann $\rho$-invariants of $\mathrm{J}$ Cheeger and M Gromov. In addition, the ranks of the above modules are new homology cobordism invariants. A common example of this Corollary is the case of the exteriors of concordant links.

Corollary 3.3 If $L_{0}$ and $L_{1}$ are compact subsets of $S^{n}$ that are concordant (or even merely $I$-equivalent) via the subset $W$ of $S^{n} \times[0,1]$ and if $A, B$ and $C$ denote the fundamental groups of the exteriors $S^{n}-L_{0}, S^{n}-L_{1}$ and $S^{n} \times[0,1]-W$ respectively then, for any $n$, the inclusion maps induce monomorphisms $A / A_{H}^{(n)} \subset C / C_{H}^{(n)}$, and $B / B_{H}^{(n)} \subset C / C_{H}^{(n)}$. Moreover $A_{H}^{(n)} / A_{H}^{(n+1)}$ and 
$B_{H}^{(n)} / B_{H}^{(n+1)}$ are modules of the same rank (over $\mathbb{Z}\left[A / A_{H}^{(n)}\right]$ and $\mathbb{Z}\left[B / B_{H}^{(n)}\right]$, respectively).

Proof By Alexander Duality the exterior $S^{n} \times[0,1]-W$ is a homology cobordism (or, in the case of $I$-equivalence, just a homology product) between the exteriors of $L_{0}$ and $L_{1}$ and so Theorem 3.1 applies.

As above, Harvey uses Corollary 3.3 to give other new results in link concordance [11. For example, note that the ranks of the above modules are new concordance invariants of links, generalizing the well-known fact that the rank of the Alexander module of a link is an invariant of concordance. It was this particular application that first motivated Harvey's definition of the torsion-free derived series. The above-mentioned Cheeger-Gromov invariants are used in 11 to show that the concordance group of disk links in any odd dimension has infinite rank even modulo local knotting.

Stallings theorem also gives a beautiful criterion to establish that a set of elements of a group generates a free subgroup. We have our own version, although we do not know an example where it is stronger than Stallings result and there are examples where it is weaker.

Proposition 3.4 If $G$ is a finitely-presented group with $H_{2}(G ; \mathbb{Q})=0$, and $x_{1}, \ldots, x_{k}$ is a set of elements of $G$ that is linearly independent in the abelianization of $G$ then this set generates a free subgroup in $G / G_{H}^{(\omega)}$.

Proof Following Stallings, let $F$ be a free group of rank $k$ equipped with the obvious map into $G$ determined by the $x_{i}$. Then apply Corollary 4.5. The result then follows easily since for the free group $F_{H}^{(\omega)} \cong F^{(\omega)}=0$.

\section{Proof of the Main Theorem}

In this section we prove our main result, Theorem 4.1 which is a direct analogue, for the torsion-free derived series, of Stallings' Rational Theorem for the rational lower central series.

Theorem 4.1 Let $A$ be finitely-generated and $B$ finitely related. Suppose $\phi: A \rightarrow B$ induces a monomorphism on $H_{1}(-; \mathbb{Q})$ and an epimorphism on $H_{2}(-; \mathbb{Q})$. Then, for each $n \leq \omega, \phi$ induces a monomorphism $A / A_{H}^{(n)} \subset B / B_{H}^{(n)}$. Moreover, if $\phi$ induces an isomorphism on $H_{1}(-; \mathbb{Q})$ then, for any finite $n$, then 
$A_{H}^{(n)} / A_{H}^{(n+1)} \rightarrow B_{H}^{(n)} / B_{H}^{(n+1)}$ is a monomorphism between modules of the same rank (over $\mathbb{Z}\left[A / A_{H}^{(n)}\right]$ and $\mathbb{Z}\left[B / B_{H}^{(n)}\right]$, respectively). If, in addition, $\phi$ is onto, then for each $n \leq \omega$ it induces an isomorphism $A / A_{H}^{(n)} \cong B / B_{H}^{(n)}$. In the case that $(B, A)$ admits the structure of a relative $2-$ complex, the first and third conclusions above remain valid without the finiteness assumptions on $A$ and $B$.

Proof of Theorem 4.1 The proof of the first claim is by induction on $n$. The case $n=1$ is clear since $A / A_{H}^{(1)}$ is merely $H_{1}(A ; \mathbb{Z}) /\{\mathbb{Z}$-Torsion $\}$ and the hypothesis that $\phi$ induces a monomorphism on $H_{1}(-; \mathbb{Q})$ implies that it also induces a monomorphism on $H_{1}(-; \mathbb{Z})$ modulo torsion. Now assume that the first claim holds for $n$, ie, $\phi$ induces a monomorphism $A / A_{H}^{(n)} \subset B / B_{H}^{(n)}$. We will prove that it holds for $n+1$.

It follows from Proposition 2.3 that $\phi\left(A_{H}^{(n+1)}\right) \subset B_{H}^{(n+1)}$. Hence the diagram below exists and is commutative. In light of the Five Lemma, we see that it suffices to show that $\phi$ induces a monomorphism $A_{H}^{(n)} / A_{H}^{(n+1)} \rightarrow B_{H}^{(n)} / B_{H}^{(n+1)}$.

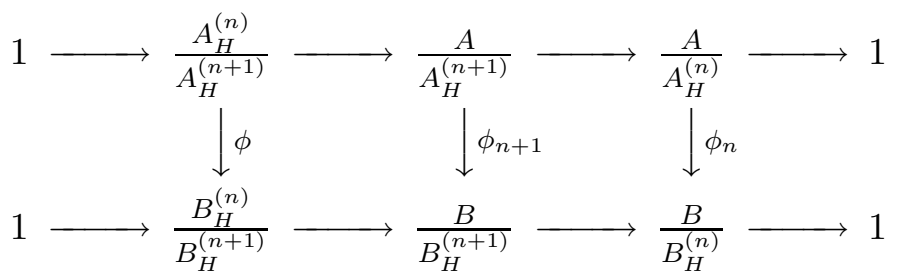

For simplicity we abbreviate $A / A_{H}^{(n)}$ by $A_{n}$ and $B / B_{H}^{(n)}$ by $B_{n}$. The inductive hypothesis is that $\phi$ induces a monomorphism $A_{n} \rightarrow B_{n}$ and hence a ring monomorphism $\mathbb{Z} A_{n} \rightarrow \mathbb{Z} B_{n}$. Since $A_{n}$ and $B_{n}$ are PTFA groups by Proposition 2.1, the rings $\mathbb{Z} A_{n}$ and $\mathbb{Z} B_{n}$ are right Ore domains and so admit classical right rings of quotients $\mathcal{K}\left(A_{n}\right)$ and $\mathcal{K}\left(B_{n}\right)$, respectively. Hence $\phi$ induces a monomorphism $\mathcal{K}\left(A_{n}\right) \rightarrow \mathcal{K}\left(B_{n}\right)$, which endows $\mathcal{K}\left(B_{n}\right)$ with the structure of a $\mathcal{K}\left(A_{n}\right)-\mathcal{K}\left(B_{n}\right)$ bimodule.

Suppose that $A_{H}^{(n)} / A_{H}^{(n+1)} \rightarrow B_{H}^{(n)} / B_{H}^{(n+1)}$ were not injective. By examining the diagram below, we see that there exists an $a \in A_{H}^{(n)}$ representing a non-torsion class $[a]$ in $A_{H}^{(n)} /\left[A_{H}^{(n)}, A_{H}^{(n)}\right]$ such that $\phi(a)$ represents a $\mathbb{Z} B_{n}$-torsion class, $[\phi(a)]$, in $B_{H}^{(n)} /\left[B_{H}^{(n)}, B_{H}^{(n)}\right]$.

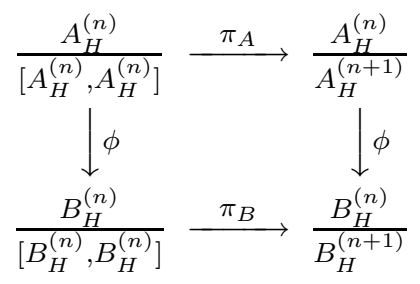


Now consider the following commutative diagram where the horizontal equivalences follow from Remark 2.12 .

$$
\begin{aligned}
& \frac{A_{H}^{(n)}}{\left[A_{H}^{(n)}, A_{H}^{(n)}\right]} \\
& \downarrow i \\
& \frac{A_{H}^{(n)}}{\left[A_{H}^{(n)}, A_{H}^{(n)}\right]} \otimes_{\mathbb{Z} A_{n}} \mathcal{K} A_{n} \\
& \downarrow \text { id } \otimes \phi \\
& \frac{A_{H}^{(n)}}{\left[A_{H}^{(n)}, A_{H}^{(n)}\right]} \otimes_{\mathbb{Z} A_{n}} \mathcal{K} B_{n} \stackrel{\cong}{\longrightarrow} H_{1}\left(A ; \mathbb{Z} A_{n}\right) \otimes_{\mathbb{Z} A_{n}} \mathcal{K} B_{n} \\
& \downarrow \phi \otimes \text { id } \quad \downarrow \phi \otimes i d \\
& \frac{B_{H}^{(n)}}{\left[B_{H}^{(n)}, B_{H}^{(n)}\right]} \otimes_{\mathbb{Z} B_{n}} \mathcal{K} B_{n} \stackrel{\cong}{\longrightarrow} H_{1}\left(B ; \mathbb{Z} B_{n}\right) \otimes_{\mathbb{Z} B_{n}} \mathcal{K} B_{n}
\end{aligned}
$$

We assert (and shall establish below) that the kernel of the vertical composition $\psi=(\phi \otimes \mathrm{id}) \circ(\mathrm{id} \otimes \phi) \circ i$ is the $\mathbb{Z} A_{n}$-torsion submodule of $A_{H}^{(n)} /\left[A_{H}^{(n)}, A_{H}^{(n)}\right]$. Assuming this, we finish the inductive proof. Note that, $\psi([a])$ is $[\phi(a)] \otimes 1$. Since $[\phi(a)]$ is $\mathbb{Z} B_{n}$-torsion, $\psi([a])=0$, since tensoring with the quotient field kills the torsion submodule [26, Corollary II.3.3]. By the assertion, [a] is $\mathbb{Z} A_{n}$-torsion, contradicting the choice of $[a]$. This contradiction shows that $A_{H}^{(n)} / A_{H}^{(n+1)} \rightarrow B_{H}^{(n)} / B_{H}^{(n+1)}$ is injective. The injectivity at the first infinite ordinal follows immediately, finishing the inductive step of the proof of the first part of Theorem 4.1, modulo our assertion.

Now we set out to establish that the kernel of $\psi$ is the $\mathbb{Z} A_{n}$-torsion submodule of $A_{H}^{(n)} /\left[A_{H}^{(n)}, A_{H}^{(n)}\right]$. The kernel of $i$ is precisely this submodule since tensoring with the quotient field kills precisely the torsion submodule. Therefore it suffices to show that the other two maps in the composition are injective.

First note that id $\otimes \phi$ is injective by an application of the following Lemma 4.2

Lemma 4.2 Suppose $H \subset G$ and $\mathbb{Z} H$ and $\mathbb{Z} G$ are Ore Domains. Then for any right $\mathbb{Z} H$-module $M$, the map

$$
i d \otimes i: M \otimes_{\mathbb{Z} H} \mathcal{K} H \rightarrow M \otimes_{\mathbb{Z} H} \mathcal{K} G
$$

is a monomorphism (of right $\mathcal{K} H$-modules). Moreover, the $\mathcal{K} H$-rank of the domain of this map equals the $\mathcal{K} G$-rank of the range.

Proof of Lemma 4.2 Since $\mathcal{K} H$ is a $\mathbb{Z} H-\mathcal{K} H$ bimodule, $M \otimes_{\mathbb{Z} H} \mathcal{K} H$ is a right $\mathcal{K} H$-module. Since any $\mathcal{K} H$-module is free [26. Proposition I.2.3], there 
is some index set $I$ such that $\psi: M \otimes_{\mathbb{Z} H} \mathcal{K} H \cong \oplus_{I} \mathcal{K} H$ as right $\mathcal{K} H$-modules. Thus the $\mathcal{K} H$-rank of the domain of $i d \otimes i$ is the cardinality of $I$. Hence

$$
\psi \otimes \mathrm{id}:\left(M \otimes_{\mathbb{Z} H} \mathcal{K} H\right) \otimes_{\mathcal{K} H} \mathcal{K} G \longrightarrow\left(\oplus_{I} \mathcal{K} H\right) \otimes_{\mathcal{K} H} \mathcal{K} G
$$

is an isomorphism of right $\mathcal{K} G$-modules, and hence of right $\mathcal{K} H$-modules. Since the domain of $\psi \otimes$ id is isomorphic to $M \otimes_{\mathbb{Z} H} \mathcal{K} G$, we can use $\psi$ and $\psi \otimes$ id to see that the first part of the Lemma is equivalent to showing that $\oplus_{I} \mathcal{K} H \longrightarrow\left(\oplus_{I} \mathcal{K} H\right) \otimes_{\mathcal{K} H} \mathcal{K} G$ is a monomorphism. But, after identifying the latter with $\oplus_{I} \mathcal{K} G$, this map is just the inclusion $\mathcal{K} H \subset \mathcal{K} G$ on each coordinate and is thus injective. Moreover this shows that the $\mathcal{K} G$-rank of $M \otimes_{\mathbb{Z} H} \mathcal{K} G$ is also equal to the cardinality of $I$.

Finally, returning to the proof of our assertion, we claim that the map, $\phi \otimes$ id, shown on the right hand side of the diagram above, is also injective. This will follow immediately from Proposition 4.3 below (setting $\Gamma=B_{n}$ ), once we identify the domain of $\phi \otimes$ id with $H_{1}\left(A ; \mathcal{K} B_{n}\right)$ and its range with $H_{1}\left(B ; \mathcal{K} B_{n}\right)$. The latter is immediate since we have previously observed that $\mathcal{K} B_{n}$ is a flat $\mathbb{Z} B_{n}$-module. For the former, note that, since any module over a division ring is free, $\mathcal{K} B_{n}$ is a free, and hence flat, $\mathcal{K} A_{n}$ module. Moreover $\mathcal{K} A_{n}$ is a flat $\mathbb{Z} A_{n}$-module. Hence $H_{1}\left(A ; \mathcal{K} B_{n}\right) \cong H_{1}\left(A ; \mathbb{Z} A_{n}\right) \otimes_{\mathbb{Z} A_{n}} \mathcal{K} B_{n}$. This completes the proof of the first claim of Theorem 4.1. modulo the proof of Proposition 4.3.

The following proposition is an important result in its own right. In the case that $\phi: A \rightarrow B$ induces an isomorphism on $H_{1}(-; \mathbb{Q})$, this result was proved in [6. Proposition 2.10]. The more general result below seems to require a different proof. Here, by $\mathcal{K} \Gamma$ we mean the right ring of fractions of the Ore domain $\mathbb{Z} \Gamma$. Recall that any homomorphism $\psi: B \rightarrow \Gamma$ endows $\mathbb{Z} \Gamma$ and $\mathcal{K} \Gamma$ with left $\mathbb{Z} B$ module structures. We call such a $\psi$ a coefficient system on $B$. If $\phi: A \rightarrow B$ then $\mathcal{K} \Gamma$ acquires a left $\mathbb{Z} A$-module structure via $\psi \circ \phi$.

Proposition 4.3 Suppose $\phi: A \rightarrow B$ induces a monomorphism (respectively, an isomorphism) on $H_{1}(-; \mathbb{Q})$ and an epimorphism on $H_{2}(-; \mathbb{Q})$. Suppose also that $A$ is finitely generated and $B$ is finitely related. Then for any coefficient system $\psi: B \rightarrow \Gamma$, where $\Gamma$ is a PTFA group, $\phi$ induces a monomorphism (respectively, an isomorphism) $H_{1}(A ; \mathcal{K} \Gamma) \rightarrow H_{1}(B ; \mathcal{K} \Gamma)$. Moreover, if the pair of Eilenberg-Maclane spaces $(K(B, 1), K(A, 1))$ has the homotopy type of a relative 2-complex, then the finiteness assumptions on $A$ and $B$ are not necessary to get a monomorphism $H_{1}(A ; \mathcal{K} \Gamma) \rightarrow H_{1}(B ; \mathcal{K} \Gamma)$.

Before proving Proposition 4.3, we finish the proof of the rest of the parts of our main theorem. 
Note that the finiteness assumptions on $A$ and $B$ are used only in the application of Proposition 4.3. Thus, if the pair of Eilenberg-Maclane Spaces $(K(B, 1), K(A, 1))$ has the homotopy type of a relative 2 -complex, then we do not need these finiteness assumptions to deduce the first part of the theorem.

Now, assume that $\phi$ induces an isomorphism on $H_{1}(-; \mathbb{Q})$. We must show that $A_{H}^{(n)} / A_{H}^{(n+1)} \rightarrow B_{H}^{(n)} / B_{H}^{(n+1)}$ is a monomorphism between modules of the same rank. The fact that this is a monomorphism follows from the first part of the theorem. Since $A_{H}^{(n)} / A_{H}^{(n+1)}$ and $A_{H}^{(n)} /\left[A_{H}^{(n)}, A_{H}^{(n)}\right]$ differ only by $\mathbb{Z} A_{n}-$ torsion, they have the same rank, $r_{A}$, as $\mathbb{Z} A_{n}$-modules. For the same reason, $A_{H}^{(n)} / A_{H}^{(n+1)} \otimes_{\mathbb{Z} A_{n}} \mathcal{K}\left(B_{n}\right)$ and $A_{H}^{(n)} /\left[A_{H}^{(n)}, A_{H}^{(n)}\right] \otimes_{\mathbb{Z} A_{n}} \mathcal{K}\left(B_{n}\right)$ are isomorphic. By Lemma 4.2 the former has $\mathcal{K}\left(B_{n}\right)$-rank equal to $r_{A}$ and hence so does the latter, which we have identified with $H_{1}\left(A ; \mathcal{K}\left(B_{n}\right)\right)$. If $\phi$ induces an isomorphism on $H_{1}(-; \mathbb{Q})$ then note that $B$ must be finitely generated. Hence Proposition 4.3 applies to show that $H_{1}\left(A ; \mathcal{K}\left(B_{n}\right)\right) \cong H_{1}\left(B ; \mathcal{K}\left(B_{n}\right)\right)$. Thus the latter has $\mathcal{K}\left(B_{n}\right)$-rank equal to $r_{A}$. But by applying the same reasoning as above, we see that it has $\mathcal{K}\left(B_{n}\right)$-rank equal to $r_{B}$, the $\mathbb{Z} B_{n}$-rank of $B_{H}^{(n)} / B_{H}^{(n+1)}$.

If $\phi$ is also onto then it induces epimorphisms on all the quotients by the terms of the torsion-free derived series. This combined with the first part of the theorem implies that it induces isomorphisms on all these quotients for $n \leq \omega$. Note also that since this argument does not use the argument of the preceding paragraph, it holds without the finiteness assumptions on $A$ and $B$ if $(\mathrm{B}, \mathrm{A})$ is 2 -dimensional in the sense described.

This concludes the proof of our main theorem, modulo the proof of Proposition 4.3

Proof of Proposition 4.3 We need the following extension of a result of Ralph Strebel.

Lemma 4.4 Suppose $\tilde{f}: M \rightarrow N$ is a homomorphism between free $\mathbb{Z} \Gamma$ modules with $\Gamma$ PTFA and let $f=\tilde{f} \otimes$ id be the induced homomorphism of abelian groups $M \otimes_{\mathbb{Z} \Gamma} \mathbb{Z} \rightarrow N \otimes_{\mathbb{Z} \Gamma} \mathbb{Z}$. Then $\operatorname{rank}_{\mathcal{K} \Gamma}($ image $\tilde{f}) \geq \operatorname{rank}_{\mathbb{Q}}($ image $f)$.

In 27. page 305], Strebel shows that, under the hypotheses of Lemma 4.4 if $f$ is injective then $\tilde{f}$ is injective. There he shows that the class, $\mathcal{D}(\mathbb{Z})$, of groups $\Gamma$ for which this property is satisfied includes torsion-free abelian groups and is closed under various natural operations such as extensions. Consequently, any PTFA group is in this class. This class of groups was previously called conservative and was later shown by J Howie and H Schneebli to coincide with 
the class of locally indicable groups 14. Our lemma shows, in the case that $M$ is finitely generated, that more generally the rank of the kernel of $\tilde{f}$ is at most the rank of the kernel of $f$.

Proof of Lemma 4.4 By the rank of a homomorphism we shall mean the rank of its image. Suppose that $\operatorname{rank}_{\mathbb{Q}} f \geq r \leq \infty$. Then there is a monomorphism $g: \mathbb{Z}^{r} \rightarrow N \otimes_{\mathbb{Z} \Gamma} \mathbb{Z}$ whose image is a subgroup of image $f$. If $e_{i}, 1 \leq i \leq r$ is a basis of $\mathbb{Z}^{r}$, choose $M_{i} \in M \otimes_{\mathbb{Z} \Gamma} \mathbb{Z}$ such that $f\left(M_{i}\right)=g\left(e_{i}\right)$. Since the "augmentation" $\epsilon_{M}: M \rightarrow M \otimes_{\mathbb{Z} \Gamma} \mathbb{Z}$ is surjective there exist elements $m_{i} \in M$ such that $\epsilon_{M}\left(m_{i}\right)=M_{i}$. Consider the map $\tilde{g}:(\mathbb{Z} \Gamma)^{r} \rightarrow N$ defined by sending the $i^{\text {th }}$ basis element to $\tilde{f}\left(m_{i}\right)$. The augmentation of $\tilde{g}, \tilde{g} \otimes \mathrm{id}$, is the map $(\mathbb{Z} \Gamma)^{r} \otimes_{\mathbb{Z} \Gamma} \mathbb{Z} \rightarrow N \otimes_{\mathbb{Z} \Gamma} \mathbb{Z}$ that sends $e_{i}$ to $\epsilon_{N}\left(\tilde{f}\left(m_{i}\right)\right)=f\left(\epsilon_{M}\left(m_{i}\right)\right)=g\left(e_{i}\right)$ and thus is seen to be identifiable with $g$. In particular $\tilde{g} \otimes$ id is a monomorphism, and thus by [27, page 305] (as mentioned above), $\tilde{g}$ is a monomorphism. Since the image of $\tilde{g}$ lies in the image of $\tilde{f}, \tilde{g}$ yields a monomorphism from $(\mathbb{Z} \Gamma)^{r}$ into the image of $\tilde{f}$, showing that the rank of image $\tilde{f}$ is at least $r$.

Now we continue with the proof of Proposition 4.3. We can find connected CW-complexes $X_{A}, X_{B}$ such that $\pi_{1}\left(X_{A}\right) \cong A$ and $\pi_{1}\left(X_{B}\right) \cong B$ and whose universal covers are contractible (classifying spaces for $A, B)$. We can find a cellular map $h: X_{A} \rightarrow X_{B}$ inducing $\phi$ on $\pi_{1}$, and by replacing $X_{B}$ by the mapping cylinder of $h$, we may assume that $h$ embeds $X_{A}$ as a subspace of $X_{B}$. The finiteness hypotheses on $A$ and $B$ are designed to ensure that (by proper choice of the cell structure on $\left.X_{A}, X_{B}\right)$ we may assume that the relative cellular chain group $C_{2}\left(X_{B}, X_{A}\right)$ (respectively, $C_{2}\left(X_{B}, X_{A}\right)$ and $C_{1}\left(X_{B}, X_{A}\right)$ ) has finite rank. (Note that if $\phi$ induces an isomorphism on $H_{1}(-; \mathbb{Q})$ then $B$ must be finitely presented). The coefficient systems $\psi$ and $\psi \circ \phi$ induce covering spaces $\widetilde{X}_{B}$ and $\widetilde{X}_{A}$ equipped with induced $\Gamma$-actions (these principal $\Gamma$-bundles are connected covering spaces in case $\psi$ and $\psi \circ \phi$ are surjective). We can lift the cell structure and consider the relative free $\mathbb{Z} \Gamma$-chain complex $C_{*}\left(\widetilde{X}_{B}, \widetilde{X}_{A}\right)$ where $C_{p}\left(\widetilde{X}_{B}, \widetilde{X}_{A}\right)$ has $\mathbb{Z} \Gamma$-rank equal to the $\mathbb{Z}$-rank of $C_{p}\left(X_{B}, X_{A}\right)$ and where the projection maps $\pi_{p}: C_{p}\left(\widetilde{X}_{B}, \widetilde{X}_{A}\right) \rightarrow C_{p}\left(X_{B}, X_{A}\right)$ commute with the $\partial$ maps $\left(\partial_{p} \circ \pi_{p}=\pi_{p-1} \circ \tilde{\partial}_{p}\right)$. Moreover the projection map can be identified with the augmentation $C_{p}\left(\widetilde{X}_{B}, \widetilde{X}_{A}\right) \rightarrow C_{p}\left(\widetilde{X}_{B}, \widetilde{X}_{A}\right) \otimes_{\mathbb{Z} \Gamma} \mathbb{Z} \cong$ $C_{p}\left(X_{B}, X_{A}\right)$ in such a way that $\partial_{p}=\tilde{\partial}_{p} \otimes$ id. By Lemma 4.4 applied to $\tilde{\partial}_{i}: C_{i}\left(\widetilde{X}_{B}, \widetilde{X}_{A}\right) \rightarrow C_{i-1}\left(\widetilde{X}_{B}, \widetilde{X}_{A}\right)$ we have that $\operatorname{rank}_{\mathcal{K} \Gamma} \tilde{\partial}_{p} \geq \operatorname{rank}_{\mathbb{Q}} \partial_{p}$ for $p=1,2,3$. Since the hypotheses imply that $H_{2}\left(X_{B}, X_{A} ; \mathbb{Q}\right)=0$ (respectively, in addition that $\left.H_{1}\left(X_{B}, X_{A} ; \mathbb{Q}\right)=0\right)$ we see that $\operatorname{rank}_{\mathbb{Q}} H_{2}\left(C_{*}\left(X_{B}, X_{A}\right)\right)=0$ (respectively, in addition $\left.\operatorname{rank}_{\mathbb{Q}} H_{1}\left(C_{*}\left(X_{B}, X_{A}\right)\right)=0\right)$. We now claim that 
$\operatorname{rank}_{\mathcal{K} \Gamma} H_{2}\left(C_{*}\left(\widetilde{X}_{B}, \widetilde{X}_{A}\right)\right)=0$ (respectively, in addition $\operatorname{rank}_{\mathcal{K} \Gamma} H_{1}\left(C_{*}\left(\widetilde{X}_{B}, \widetilde{X}_{A}\right)\right)$ is 0$)$. This follows since (letting $c_{2}<\infty$ be the rank of $C_{2}\left(\widetilde{X}_{B}, \widetilde{X}_{A}\right)$ ):

$$
\begin{aligned}
\operatorname{rank}_{\mathcal{K} \Gamma}\left(H_{2}\left(C_{*}\left(\widetilde{X}_{B}, \widetilde{X}_{A}\right)\right)\right) & =\operatorname{rank}_{\mathcal{K} \Gamma}\left(\operatorname{ker} \tilde{\partial}_{2}\right)-\operatorname{rank}_{\mathcal{K} \Gamma} \tilde{\partial}_{3} \\
& =\left(c_{2}-\operatorname{rank}_{\mathcal{K} \Gamma} \tilde{\partial}_{2}\right)-\operatorname{rank}_{\mathcal{K} \Gamma} \tilde{\partial}_{3} \\
& \leq c_{2}-\operatorname{rank}_{\mathbb{Q}} \partial_{2}-\operatorname{rank}_{\mathbb{Q}} \partial_{3} \\
& =\operatorname{rank}_{\mathbb{Q}}\left(\operatorname{ker} \partial_{2}\right)-\operatorname{rank}_{\mathbb{Q}} \partial_{3} \\
& =\operatorname{rank}_{\mathbb{Q}}\left(H_{2}\left(C_{*}\left(X_{B}, X_{A}\right)\right)\right) \\
& =0 .
\end{aligned}
$$

A similar argument holds for $H_{1}$ when $C_{1}\left(X_{B}, X_{A}\right)$ has finite rank. Hence $H_{2}\left(\widetilde{X}_{B}, \widetilde{X}_{A}\right)$ has zero rank (respectively, in addition $H_{1}\left(\widetilde{X}_{B}, \widetilde{X}_{A}\right)$ has zero rank). But the equivariant homology modules $H_{p}\left(\widetilde{X}_{B}, \widetilde{X}_{A}\right)$ are well known to be isomorphic to the homology with coefficients in $\psi, H_{p}\left(X_{B}, X_{A} ; \mathbb{Z} \Gamma\right)[29$, Theorems VI3.4 and 3.4*]. Moreover the homology of a group $G$ is well known to be the same as that of its associated Eilenberg-Maclane space $K(G, 1)$ [13. page 335]. Thus we have shown that $H_{2}(B, A ; \mathbb{Z} \Gamma) \otimes \mathcal{K} \Gamma=H_{2}(B, A ; \mathcal{K} \Gamma)=0$ (respectively, in addition $H_{1}(B, A ; \mathcal{K} \Gamma)=0$ ) and consequently $\phi$ induces a monomorphism (respectively an isomorphism) $\phi: H_{1}(A ; \mathcal{K} \Gamma) \rightarrow H_{1}(B ; \mathcal{K} \Gamma)$ as desired.

If we do not have the finiteness assumptions, but $\left(X_{B}, X_{A}\right)$ is a 2-complex, we can show that $H_{2}(B, A ; \mathbb{Q} \Gamma)=0$ by a direct application of Strebel's Lemma. Specifically, the hypothesis that $H_{2}(B, A ; \mathbb{Q})=0$ implies that the map $\partial_{2}$ : $C_{2}\left(X_{B}, X_{A} ; \mathbb{Q}\right) \rightarrow C_{1}\left(X_{B}, X_{A} ; \mathbb{Q}\right)$ is injective. By Strebel's result, $\tilde{\partial}_{2}$ is injective and the claimed result follows. Hence, in this case even without the finiteness assumptions, we deduce that $\phi: H_{1}(A ; \mathcal{K} \Gamma) \rightarrow H_{1}(B ; \mathcal{K} \Gamma)$ is injective.

Corollary 4.5 Let $F$ be a free group and $B$ be finitely related with $H_{2}(B ; \mathbb{Q})$ $=0$. Suppose $\phi: F \rightarrow B$ induces a monomorphism on $H_{1}(-; \mathbb{Q})$. Then, for any finite $n$, it induces monomorphisms $F / F^{(n)} \subset B / B^{(n)}$.

Proof of Corollary 4.5 Let $F$ be a finitely generated free group. Thus, by Theorem 4.1] $F / F^{(n)} \hookrightarrow B / B_{H}^{(n)}$. Since $B^{(n)} \subset B_{H}^{(n)}$, we see that $F / F^{(n)} \hookrightarrow$ $B / B^{(n)}$. Then we only need comment on why it is not necessary to require that $F$ be finitely generated. If it is not finitely generated, and if for some fixed $n$, the map $F / F^{(n)} \longrightarrow B / B^{(n)}$ has a nontrivial element $w$ in its kernel, then $w$ is represented by a finite word which thus lies in a finite rank free subgroup $G$ of $F$ (free on the letters that appear in $w$ ). Then $\phi$ induces a map $\phi: G \rightarrow B$ 
that is a monomorphism on $H_{1}(-; \mathbb{Q})$ and for which the induced map the map $G / G^{(n)} \longrightarrow B / B^{(n)}$ has a nontrivial kernel, contradicting Theorem 4.1 .

Remark 4.6 The first and second parts of Theorem 4.1 cannot be improved to have isomorphisms in the conclusion, even for integral homology equivalences. For there exist finitely presented groups $E$ and $\phi: F\langle x, y\rangle \rightarrow E$ inducing isomorphisms on all integral homology groups but where the induced map:

$$
\frac{F_{H}^{(1)}}{F_{H}^{(2)}} \cong \frac{F^{(1)}}{F^{(2)}} \longrightarrow \frac{E^{(1)}}{E^{(2)}} \cong \frac{E_{H}^{(1)}}{E_{H}^{(2)}}
$$

is not surjective. One such example is $E=\left\langle t, w, z \mid t=z^{3} w^{2} t w^{-1} z^{-3}\right\rangle$ and $F=$ $F\langle t, z\rangle$. Then $F^{(1)} / F^{(2)}$ is a free $\mathbb{Z}\left[t^{ \pm 1}, z^{ \pm 1}\right]$-module of rank 1 but $E_{H}^{(1)} / E_{H}^{(2)}$ is not even a projective module. Such groups arise commonly as the fundamental groups of the exteriors in $B^{4}$ of a set of ribbon disks for a ribbon link.

Remark 4.7 Theorem 4.1 can fail if $A$ is not finitely generated. Let $A=$ $\left\langle x, w_{i}, i \in \mathbb{Z} \mid w_{i}=\left[x^{-1}, w_{i+1}\right]\right\rangle$ and $B=\mathbb{Z}$. The abelianization $\phi: A \rightarrow B$ induces an isomorphism on $H_{1}(-; \mathbb{Z})$ and an epimorphism on $H_{2}(-; \mathbb{Z})$, but $A^{(1)} / A^{(2)}$ has rank 1 (it has a $\mathbb{Z}\left[x^{ \pm 1}\right]$-module presentation $\left\langle w_{i}, i \in \mathbb{Z}\right| w_{i}=$ $\left.\left.w_{i+1}(x-1)\right\rangle\right)$ so $\phi$ does not induce a monomorphism on $A / A_{H}^{(2)}$.

Remark 4.8 The epimorphism part of the conclusion of Proposition 4.3 can fail if $B$ is not finitely related using the same groups as above but with the roles reversed, $\phi: \mathbb{Z} \rightarrow A$. The same example shows that the part of the conclusion of Theorem 4.1]about "having the same rank" can fail if $B$ is not finitely related.

\section{Homological completions and localizations}

In this section we construct a rational homological localization functor, $G \rightarrow \widetilde{G}$, that we call the torsion-free-solvable completion. As we explain below, in the context of rational homological localization, this can be viewed as an analogue of the Malcev completion wherein one replaces the lower central series by the torsion-free derived series. The latter is quite a bit more complicated because whereas the lower-central series quotients $G_{n} / G_{n+1}$ are trivial modules (over $\left.G / G_{n}\right)$, their analogues $G^{(n)} / G^{(n+1)}$ are not. We parallel A. Bousfield's discussion of the Malcev completion (also called Malcev localization) [1] and other homological localizations. At the end of the section we also compare our localization to the universal (integral) homological localization functor due to $\mathrm{P}$ Vogel and J Levine. 
We remark that, in the context of linear algebraic groups, C Miller and R Hain have defined a "relative solvable completion" [20, 10]. This notion differs from ours. It is related to the lower-central series of the commutator subgroup. In particular, it is not invariant under homological equivalence. We also note that, subsequent to our work, Jae Choon Cha has shown that our $\widetilde{G}$ is not the "initial functor" satisfying (1) and (2) below and has identified the initial such functor 4.

Theorem 5.1 For any group $G$ there is a group $\widetilde{G}$ and a homomorphism $f: G \rightarrow \widetilde{G}$ such that:

(1) $\operatorname{ker} f=G_{H}^{(w)}$

(2) If $A$ is finitely generated, $B$ is finitely-presented and $\phi: A \rightarrow B$ induces an isomorphism (respectively, a monomorphism) on $H_{1}(-; \mathbb{Q})$ and an epimorphism on $\mathrm{H}_{2}(-; \mathbb{Q})$ then there is an isomorphism (respectively, monomorphism) $\tilde{\phi}: \widetilde{A} \rightarrow \widetilde{B}$ such that the following commutes:

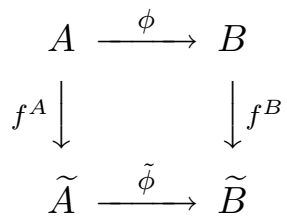

Before proceeding we review the Malcev completion so that the reader can see the analogy to the torsion-free-solvable completion. Recall that a nilpotent group $N$ is a uniquely divisible nilpotent group if, for every positive integer $m$, the function $N$ to $N$ given by $x \rightarrow x^{m}$, is a bijection [1, page 3]. (If $\bigcap_{n=1}^{\infty} N_{n}^{r}=0$ then this can be shown to be equivalent to requiring that the quotients, $N_{n}^{r} / N_{n+1}^{r}$, of successive terms of the rational lower central series are uniquely divisible $\mathbb{Z}$-modules). For a nilpotent group, $N$, the Malcev completion $N \rightarrow \operatorname{MC}(N)$, usually (sloppily) denoted $N \otimes \mathbb{Q}$, is a uniquely divisible nilpotent group such that $N \rightarrow N \otimes \mathbb{Q}$ is initial among maps to uniquely divisible nilpotent groups [1, page 3] [23] 21, Proposition 3.3]. This is identical to Bousfield's $H \mathbb{Q}$-completion [1, Proposition 1.6]. More intuitively, $N \otimes \mathbb{Q}$ is obtained from the trivial group by successive central extensions by the vector spaces $\left(N_{i}^{r} / N_{i+1}^{r}\right) \otimes \mathbb{Q}$ where the extensions are "compatible" with those that define $N$. For a general group , $G$, the Malcev completion $M C(G)$ is $\lim M C\left(G / G_{n}\right)$ or, equivalently, $\lim M C\left(G / G_{n}^{r}\right)$ (the $\mathbb{Q}$-completion of $G$ in Bousfield's language [1, page 16]). Note that, by Stallings' Integral Theorem, the nilpotent completion, $\lim G / G_{n}$, is invariant under integral homology equivalence of groups but is not invariant under rational homology equivalence. Even 
the rational nilpotent completion, $\lim G / G_{n}^{r}$, is not an invariant of rational homology equivalence (see Stallings' Rational Theorem). But the Malcev completion of $G$ is invariant under rational homology equivalence (as are each of the groups $\left.M C\left(G / G_{n}\right)\right)$ as indicated by the last part of Stallings' Rational Theorem.

Theorem 5.1 will be proved by directly constructing an $n$-solvable version of $\widetilde{G}$ called $\widetilde{G}_{n}$ and then setting $\widetilde{G}=\lim \widetilde{G}_{n}$ analogous to the construction of the Malcev/Bousfield completion. By way of further analogy, we will see that the torsion-free-solvable completion of a group $N$ is obtained from the trivial group successive extensions by the tensor product of the modules $N_{H}^{(i)} / N_{H}^{(i+1)}$ with appropriate skew fields. The torsion-free-solvable completion, $\widetilde{G}$, and the individual groups $\left(\widetilde{G / G_{H}^{(n)}}\right)$ will be seen to be invariant under rational homology equivalence. However, we have been unable to prove the result, which we expect is true, that our $\widetilde{G}_{n}$ is initial in the appropriate sense (among functors satisfying the properties of Theorem 5.6. Without this fact, the analogy to the Malcev completion is incomplete. The problem seems to be a failure of functoriality (since the torsion-free-derived series is not fully invariant). The authors expect this to be repaired by the modifications of Remark 5.22 .

Definition 5.2 A group $A$ is $n$-torsion-free-solvable if $A_{H}^{(n)}=0$ and is torsionfree-solvable if it is $n$-torsion-free-solvable for some integer $n$.

Note that any torsion-free-solvable group $N$ is obtained from the trivial group by successive extensions by the torsion-free modules $N_{H}^{(i)} / N_{H}^{(i+1)}$. In particular, any such group is poly-(torsion-free-abelian).

Definition 5.3 (Compare [1, Section 12]) A collection of groups $A_{n}, n \geq 0$, and group homomorphisms $f_{n}, \pi_{n}, n \geq 0$, as below:

$$
A \stackrel{f_{n}}{\longrightarrow} A_{n} \stackrel{\pi_{n}}{\longrightarrow} A_{n-1}
$$

compatible in the sense that $f_{n-1}=\pi_{n} \circ f_{n}$, is a torsion-free-solvable tower for $A$ if, for each $n, A_{n}$ is $n$-torsion-free-solvable and the kernel of $\pi_{n}$ is contained in $\left(A_{n}\right)_{H}^{(n-1)}$. A truncated tower, $A_{n} \rightarrow \ldots \rightarrow A_{0}$, is called a tower of height $n$.

Definition 5.4 A right module $M$ over an integral domain $R$ is a (uniquely) divisible $R$-module if, for each $m \in M$ and non-zero $r \in R$, there exists some (unique) $m^{\prime} \in M$ such that $m=m^{\prime} r$. 
Definition 5.5 A torsion-free-solvable group $A$ is a (uniquely) divisible torsion-free-solvable group if, for each $n, A_{H}^{(n)} / A_{H}^{(n+1)}$ is a (uniquely) divisible $\mathbb{Z}\left[A / A_{H}^{(n)}\right]$-module.

Theorem 5.6 For any group $G$ there exists a torsion-free-solvable tower, $\left\{\widetilde{G}_{n}, f_{n}: G \rightarrow \widetilde{G}_{n}, \pi_{n}: \widetilde{G}_{n} \rightarrow \widetilde{G}_{n-1}\right\}$ such that:

(1) $\operatorname{ker} f_{n}=G_{H}^{(n)}$

(2) $\widetilde{G}_{n}$ is a uniquely divisible $n$-torsion-free-solvable group.

(3) If $A$ is finitely generated, $B$ is finitely presented and $\phi: A \rightarrow B$ induces an isomorphism (respectively, monomorphism) on $H_{1}(-; \mathbb{Q})$ and an epimorphism on $H_{2}(-; \mathbb{Q})$ then there is an isomorphism (respectively, monomorphism) $\tilde{\phi}_{n}: \widetilde{A}_{n} \rightarrow \widetilde{B}_{n}$ such that the following commutes:

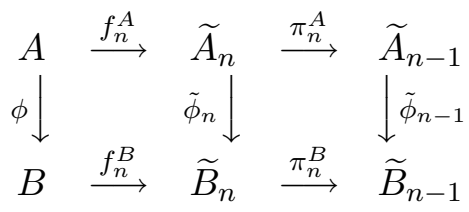

(4) $\widetilde{G}_{n}$ depends only on $G / G_{H}^{(n)}$, that is if $\phi: A \rightarrow B$ induces an isomorphism $A / A_{H}^{(m)} \cong B / B_{H}^{(m)}$ then it induces an isomorphism $\tilde{\phi}: \widetilde{A}_{n} \rightarrow \widetilde{B}_{n}$ for all $n \leq m$. In particular, $\widetilde{G}_{n} \cong\left(\widetilde{G / G_{H}^{(m)}}\right)_{n}$ if $n \leq m$.

Proof that Theorem $\mathbf{5 . 6}$ implies Theorem 5.1 Let $\widetilde{G}=\underset{\lim }{5} \widetilde{G}_{n}$ and let $f: G \rightarrow \widetilde{G}$ be the map induced by the collection of $f_{n}: G \rightarrow \widetilde{G_{n}}$. Property 1 of Theorem 5.1 follows directly from property 1 of Theorem 5.6 .

Proof of Theorem $\underline{\mathbf{5 . 6}}$ We construct such groups $\left(\widetilde{G}_{n}, f_{n}\right)$ recursively. Set $\widetilde{G}_{0}=\{e\}$. Suppose $\widetilde{G}_{i}, \pi_{i}$ and $f_{i}$ have been defined for $0 \leq i \leq n$ in such a way that Properties $1_{n}$ and $2_{n}$ are satisfied.

We first define $\widetilde{G}_{n+1}$. Since $\widetilde{G}_{n}$ is torsion-free-solvable it is poly-torsion-freeabelian so $\mathbb{Z} \widetilde{G}_{n}$ is an Ore domain as in Proposition 2.1. Thus $\mathcal{K} \widetilde{G}_{n}$ exists and is a $\mathbb{Z} \widetilde{G}_{n}-\mathcal{K} \widetilde{G}_{n}$ bimodule. In particular $\mathcal{K} \widetilde{G}_{n}$ is a $\mathbb{Z} G-\mathbb{Z} \widetilde{G}_{n}$ bimodule via $f_{n}: G \rightarrow \widetilde{G}_{n}$ and so $H_{1}\left(G ; \mathcal{K} \widetilde{G}_{n}\right)$ is defined and has the structure of a right $\mathbb{Z} \widetilde{G}_{n}$-module. Let $\widetilde{G}_{n+1}$ be the semidirect product of $\widetilde{G}_{n}$ with $H_{1}\left(G ; \mathcal{K} \widetilde{G}_{n}\right)$. Then we have the exact sequence

$$
1 \longrightarrow H_{1}\left(G ; \mathcal{K} \widetilde{G}_{n}\right) \stackrel{i_{n}}{\longrightarrow} \widetilde{G}_{n+1} \stackrel{\pi_{n+1}}{\longrightarrow} \widetilde{G}_{n} \longrightarrow 1 .
$$


Remark 5.7 This construction is precisely analogous to Bousfield's construction of the $H \mathbb{Q}$-tower $\left\{f_{n}: G \rightarrow T_{n}, n \geq 0\right\}$ for $G$, which goes as follows 1 Section 3.4]. Given $f_{n}: G \rightarrow T_{n}$, let $V_{n}$ denote $H_{2}\left(f_{n} ; \mathbb{Q}\right)$. Then the fundamental class in $H^{2}\left(f_{n} ; V_{n}\right)$ determines a central extension

$$
1 \longrightarrow V_{n} \longrightarrow T_{n+1} \longrightarrow T_{n} \longrightarrow 1 \text {. }
$$

In our case, by analogy, given $f_{n}: G \rightarrow \widetilde{G}_{n}$, let $K$ denote $H_{2}\left(f_{n} ; \mathcal{K} \widetilde{G}_{n}\right)$ where we take into account the module structure. Now if we consider the exact sequence in homology for the pair $\left(G, \widetilde{G}_{n}\right)$ with coefficients in $\mathcal{K} \widetilde{G}_{n}$, note that $K \cong H_{1}\left(G ; \mathcal{K} \widetilde{G}_{n}\right)$ since $H_{*}\left(\widetilde{G}_{n} ; \mathcal{K} \widetilde{G}_{n}\right)=0$. Then an extension of $\widetilde{G}_{n}$ by $K$ is determined as above. In fact we shall see in Lemma 5.9 that $K$ is an injective $\mathbb{Z} \widetilde{G}_{n}$-module implying that $H^{2}\left(\widetilde{G}_{n} ; K\right)=0$, and thus that the semi-direct product is the unique such extension by $K$ [13, page 189, VI Theorem 10.3].

Next we want to show that $H_{1}\left(G ; \mathcal{K} \widetilde{G}_{n}\right)$ is a uniquely divisible $\mathbb{Z} \widetilde{G}_{n}$-module.

Lemma 5.8 If $R$ is a right Ore Domain then a torsion-free right $R$-module $M$ is (uniquely) divisible if and only if it is a (uniquely) injective module (by (uniquely) injective we mean that, for every monomorphism $\phi: L \rightarrow N$ and any homomorphism $\psi: L \rightarrow M$ there exists a (unique) extension $\psi^{\prime}: N \rightarrow M$.

Proof of Lemma 5.8 This follows from [26, Proposition 3.7 page 58, Proposition 6.5 page 21]. There Stenström does not discuss the uniqueness condition, but this is easily verified by examining the proofs.

Lemma $5.9 H_{1}\left(G ; \mathcal{K} \widetilde{G}_{n}\right)$ is a uniquely injective (and uniquely divisible) $\mathbb{Z} \widetilde{G}_{n}{ }^{-}$ module, and is a uniquely injective (and uniquely divisible) $\mathbb{Z}\left[G / G_{H}^{(n)}\right]$-module $\left(\operatorname{via} G / G_{H}^{(n)} \stackrel{f_{n}}{\longrightarrow} \widetilde{G}_{n}\right)$.

Proof of Lemma 5.9 Since $H_{1}\left(G ; \mathcal{K} \widetilde{G}_{n}\right)$ is a right $\mathcal{K} \widetilde{G}_{n}$-module and any $\mathcal{K} \widetilde{G}_{n}$-module is free, it suffices to know that $\mathcal{K} \widetilde{G}_{n}$ itself is a uniquely injective $\mathbb{Z} \widetilde{G}_{n}$-module [26, Lemma 6.4 page 20]). By Lemma [5.8 it suffices to see that $\mathcal{K} \widetilde{G}_{n}$ is uniquely divisible. But this property of a classical quotient field is trivial to check (see [26, Proposition 3.7 page 58]).

Since $f_{n}$ induces a monomorphism $\mathbb{Z}\left[G / G_{H}^{(n)}\right] \rightarrow \mathbb{Z}_{n}$ by $1_{n}, \mathcal{K}\left(G / G_{H}^{(n)}\right)$ embeds in $\mathcal{K} \widetilde{G}_{n}$. Thus the $\mathbb{Z}\left[G / G_{H}^{(n)}\right]$ structure on $H_{1}\left(G ; \mathcal{K} \widetilde{G}_{n}\right)$ factors through $\mathcal{K}\left(G / G_{H}^{(n)}\right)$. Hence $H_{1}\left(G ; \mathcal{K} \widetilde{G}_{n}\right)$ is a $\mathcal{K}\left(G / G_{H}^{(n)}\right)$-module, and therefore is isomorphic to a direct sum of copies of $\mathcal{K}\left(G / G_{H}^{(n)}\right)$. Since $\mathcal{K}\left(G / G_{H}^{(n)}\right)$ is itself a uniquely injective $\mathbb{Z}\left[G / G_{H}^{(n)}\right]$-module, as noted above (since $\mathbb{Z}\left[G / G_{H}^{(n)}\right]$ is an Ore Domain), then $H_{1}\left(G ; \mathcal{K} \widetilde{G}_{n}\right)$ is a uniquely injective $\mathbb{Z}\left[G / G_{H}^{(n)}\right]$-module. 
Now, application of the following Lemma ensures that $\widetilde{G}_{n+1}$ is a uniquely divisible $(n+1)$-torsion-free-solvable group, completing the verification of Property $2_{n+1}$.

Lemma 5.10 Suppose $B=A \rtimes C$ where $A$ is a uniquely divisible $\mathbb{Z} C$-module and $C$ is $n$-torsion-free-solvable. Then $B$ is $(n+1)$-torsion-free-solvable and $A=B_{H}^{(n)}$. If $C$ is a uniquely divisible $n$-torsion-free-solvable group then $B$ is a uniquely divisible $(n+1)$-torsion-free-solvable group.

Proof of Lemma 5.10 We may assume that $n$ is the least positive integer such that $C_{H}^{(n)}=0$ since if for some $m<n, B_{H}^{(m)}=0$ then it follows that $B_{H}^{(n)}=0$. We have the split exact sequence below

$$
1 \longrightarrow A \stackrel{i}{\longrightarrow} B \stackrel{\pi}{\longrightarrow} C \longrightarrow 1 \text {. }
$$

where $s: C \rightarrow B$ is the splitting map.

First we show by induction that $\pi_{m}: B / B_{H}^{(m)} \rightarrow C / C_{H}^{(m)}$ exists and is an isomorphism for $0 \leq m \leq n$. This is trivially true for $m=0$. We assume it is true for all values at most some $m \leq n-1$ and establish it for $m+1$. Viewing $m$ as fixed, we first wish to show that $s_{m}: C / C_{H}^{(m)} \longrightarrow B / B_{H}^{(m)}$ exists and is an isomorphism. This requires a short induction. If $s_{i}, i<m$, exists and is an isomorphism then by Proposition 2.3. $s_{i+1}$ exists. Since $i+1 \leq m$, our inductive hypothesis holds and so $\pi_{i+1}$ exists and is an isomorphism. Moreover $\pi_{i+1} \circ s_{i+1}=$ id so $s_{i+1}$ is an isomorphism. This completes the inductive proof that $s_{m}$ exists and is an isomorphism. Returning to our proof that $\pi_{m+1}$ exists and is an isomorphism, choose a non-zero $c \in C_{H}^{(n-1)}$. Since $m \leq n-1$, $c \in C_{H}^{(m)}$. Then $c-1$ is a non-zero element of $\mathbb{Z} C$ and since $A$ is a divisible $\mathbb{Z} C$-module, for any $a \in A$, there is an $\alpha \in A$ such that $a=\alpha(c-1)$. When written in terms of $B$, this says that $i(a)=\left[s(c)^{-1}, i(\alpha)\right]$. Now note that since $i(A)=\operatorname{ker} \pi$ and $\pi_{m}$ is injective, $i(A) \subset B_{H}^{(m)}$. Hence $i(\alpha) \in B_{H}^{(m)}$. Since $c \in C_{H}^{(m)}$ and $s_{m}$ exists, $s(c) \in B_{H}^{(m)}$. Thus $i(a) \in B_{H}^{(m+1)}$ and so $i(A) \subset B_{H}^{(m+1)}$. By Proposition 2.5] $\pi_{m+1}$ is an isomorphism. This concludes the inductive proof that $\pi_{n}: B / B_{H}^{(n)} \cong C / C_{H}^{(n)}$. However since $C_{H}^{(n)}=0$, this implies that $i(A)=B_{H}^{(n)}$. Therefore $B_{H}^{(n)}$ is abelian and $i(A)=B_{H}^{(n)} /\left[B_{H}^{(n)}, B_{H}^{(n)}\right]$. This quotient is naturally a $\mathbb{Z}\left[B / B_{H}^{(n)}\right]$-module (via conjugation as usual) and the action factors through the isomorphism $\pi_{n-1}: \mathbb{Z}\left[B / B_{H}^{(n)}\right] \longrightarrow \mathbb{Z}\left[C / C_{H}^{(n)}\right] \cong \mathbb{Z}[C]$ (since $i(A)$ acts trivially). By hypothesis $A$ is a uniquely divisible, hence torsion-free $\mathbb{Z} C$-module, so the submodule $B_{H}^{(n)} /\left[B_{H}^{(n)}, B_{H}^{(n)}\right]$ is a torsion-free $\mathbb{Z}\left[B / B_{H}^{(n)}\right]$ module. Then, by definition, $B_{H}^{(n+1)}=0$. 
Now suppose $C$ is a uniquely divisible $n$-torsion-free-solvable group. Since $A=B_{H}^{(n)}$, Proposition 2.5 ensures that $\pi_{m}: B_{H}^{(m-1)} / B_{H}^{(m)} \rightarrow C_{H}^{(m-1)} / C_{H}^{(m)}$ is an isomorphism for each $m \leq n$. Therefore $B_{H}^{(m-1)} / B_{H}^{(m)}$ is a uniquely divisible $\mathbb{Z}\left[C / C_{H}^{(m-1)}\right]$-module. Since $\mathbb{Z}\left[B / B_{H}^{(m-1)}\right] \cong \mathbb{Z}\left[C / C_{H}^{(m-1)}\right], B_{H}^{(m-1)} / B_{H}^{(m)}$ is a uniquely divisible $\mathbb{Z}\left[B / B_{H}^{(m-1)}\right]$-module. It remains only to verify that $B_{H}^{(n)} / B_{H}^{(n+1)}\left(=B_{H}^{(n)}=A\right)$ is a uniquely divisible $\mathbb{Z}\left[B / B_{H}^{(n)}\right]$-module. Since $\mathbb{Z}\left[B / B_{H}^{(n)}\right] \cong \mathbb{Z} C$ this is true by hypothesis.

Next we define $f_{n+1}$. Since $\widetilde{G}_{n+1}$ is a semidirect product we can specify $f_{n+1}$ uniquely by setting $f_{n+1}=\left(d_{n}, f_{n}\right)$ where $d_{n}: G \rightarrow A$ is a derivation, where we henceforth abbreviate $A=H_{1}\left(G ; \mathcal{K} \widetilde{G}_{n}\right)$, and $G$ acts (on the right) through $G \stackrel{f_{n}}{\longrightarrow} \widetilde{G}_{n}$ [13. VI Proposition 5.3]. Any such map $f_{n+1}$ will satisfy $f_{n}=$ $\pi_{n+1} \circ f_{n+1}$, so it only remains to specify $d_{n}$. To define $d_{n}$, consider the exact sequence

$$
1 \longrightarrow G_{H}^{(n)} \longrightarrow G \longrightarrow G / G_{H}^{(n)} \longrightarrow 1
$$

and the induced 5-term exact sequence [13, VI Theorem 8.1]

$$
\begin{aligned}
0 \rightarrow \operatorname{Der}\left(G / G_{H}^{(n)}, A\right) \longrightarrow & \operatorname{Der}(G, A) \stackrel{\pi}{\longrightarrow} \operatorname{Hom}_{\mathbb{Z}\left[G / G_{H}^{(n)}\right]}\left(G_{H}^{(n)} /\left[G_{H}^{(n)}, G_{H}^{(n)}\right], A\right) \longrightarrow \\
& H^{2}\left(G / G_{H}^{(n)}, A\right) \longrightarrow H^{2}(G, A) .
\end{aligned}
$$

We describe a canonical element of $\operatorname{Hom}_{\mathbb{Z}\left[G / G_{H}^{(n)}\right]}\left(G_{H}^{(n)} /\left[G_{H}^{(n)}, G_{H}^{(n)}\right], A\right)$, that we call $\left(f_{n}\right)_{*}$. Namely, consider the canonical projection $p: H_{1}\left(G ; \mathbb{Z}\left[G / G_{H}^{(n)}\right]\right) \rightarrow B$ where $B=H_{1}\left(G ; \mathbb{Z}\left[G / G_{H}^{(n)}\right]\right) /$ Torsion and the canonical injection $i: B \rightarrow B \otimes$ $\mathcal{K} \widetilde{G}_{n} \cong A$ and set $\left(f_{n}\right)_{*}=i \circ p$ viewed as a map from $G_{H}^{(n)} /\left[G_{H}^{(n)}, G_{H}^{(n)}\right]$ to $A$. Since $A$ is an injective $\mathbb{Z}\left[G / G_{H}^{(n)}\right]$-module, $H^{2}\left(G / G_{H}^{(n)} ; A\right)=0$ [13, (2.4) page 189], and so we can choose a derivation $d_{n} \in \operatorname{Der}(G, A)$ such that $\pi\left(d_{n}\right)=\left(f_{n}\right)_{*}$. Let $f_{n+1}: G \rightarrow \widetilde{G}_{n+1}$ be the induced group homomorphism. We now show that, once $f_{n}$ is fixed, $f_{n+1}$ is unique up to post-composition with an isomorphism. Suppose $f_{n+1}^{\prime}=\left(d_{n}^{\prime}, f_{n}\right)$ is another homomorphism such that $\pi\left(d_{n}^{\prime}\right)=\left(f_{n}\right)_{*}$. Then $d_{n}^{\prime}=k+d_{n}$ where $k$ is the image of an element of $\operatorname{Der}\left(G / G_{H}^{(n)}, A\right)$. Since $A$ is an injective $\mathbb{Z}\left[G / G_{H}^{(n)}\right]$ (right)-module, $H^{1}\left(G / G_{H}^{(n)}, A\right)=0$ so $k$ is the image of a principal derivation, that is, $k(g)=\alpha\left(1-g^{-1}\right)$ for some $\alpha \in A$. It is easy to verify that the automorphism $\widetilde{k}$ of $\widetilde{G}_{n+1}$ given by conjugation by $\alpha$, sends $(a, x)$ to $\left(\alpha\left(1-x^{-1}\right)+a, x\right)$. Since $f_{n+1}(g)=\left(d_{n}(g), f_{n}(g)\right)$, we see that $\widetilde{k} \circ f_{n+1}(g)$ is $\left(\alpha\left(1-f_{n}(g)^{-1}\right)+d_{n}(g), f_{n}(g)\right)=\left(k(g)+d_{n}(g), g\right)$ (since $G$ acts via $\left.f_{n}\right)$. Hence we have shown that $\left.f_{n+1}^{\prime}(g)=d_{n}^{\prime}(g), f_{n}(g)\right)=\widetilde{k} \circ f_{n+1}(g)$. Thus $f_{n+1}$ is unique up to post-composition with an automorphism of $\widetilde{G}_{n+1}$. This completes the definition of $\left\{\widetilde{G}_{n+1}, f_{n+1}\right\}$. 
Having defined $f_{n+1}$, we must verify that $\left\{\left(\widetilde{G}_{i}, \widetilde{f}_{i}\right) \mid i \leq n+1\right\}$ is indeed a torsion-free-solvable tower of height $n+1$ for $G$. Since we have shown above that $\widetilde{G}_{n+1}$ is torsion-free-solvable, we only need that $\operatorname{ker}\left(\pi_{n+1}\right) \subset\left(\widetilde{G}_{n+1}\right)_{H}^{(n)}$. But by Lemma 5.10 these groups are equal.

We can now easily see that $f_{n+1}$ satisfies $1_{n+1}$, for if $f_{n+1}(g)=(0, e)$ then $g \in \operatorname{ker} f_{n}$ and $d_{n}(g)=0$. Thus $g \in G_{H}^{(n)}$, by $1_{n}$, and $[g]$ lies in $\operatorname{ker}\left(f_{n}\right)_{*}$. But the kernel of $\left(f_{n}\right)_{*}$ is precisely the subgroup represented by $G_{H}^{(n+1)}$, by definition, so $g \in G_{H}^{(n+1)}$.

Now we verify Property 3. Suppose $\phi: A \rightarrow B$ is a homomorphism satisfying the hypotheses of Property 3 and suppose, by induction that $\phi$ induces an isomorphism (respectively, monomorphism) $\tilde{\phi}: \widetilde{A}_{n} \rightarrow \widetilde{B}_{n}$ such that the diagram below commutes

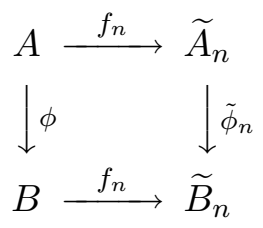

Note that the composition:

$$
H_{1}\left(A ; \mathcal{K} \widetilde{A}_{n}\right) \stackrel{\tilde{\phi}_{n}}{\longrightarrow} H_{1}\left(A ; \mathcal{K} \widetilde{B}_{n}\right) \stackrel{\phi_{n}}{\longrightarrow} H_{1}\left(B ; \mathcal{K} \widetilde{B}_{n}\right)
$$

is an isomorphism (respectively, monomorphism) of $\mathcal{K} \widetilde{A}_{n}$ modules by Proposition 4.3. This map, together with $\tilde{\phi}_{n}$, induces an isomorphism (monomorphism) of extensions.

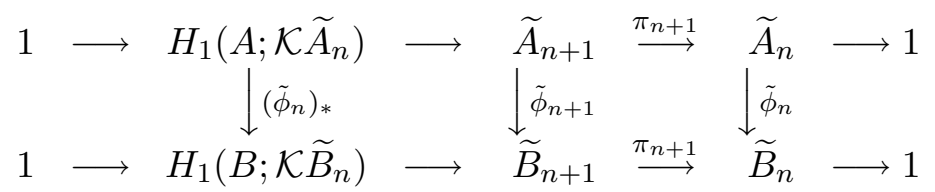

This finishes the proof of $3_{n+1}$ except for verifying the compatibility of maps. To verify that $\tilde{\phi}_{n+1} \circ f_{n+1}^{A}=f_{n+1}^{B} \circ \phi$, observe that since $\widetilde{A}_{n+1}$ and $\widetilde{B}_{n+1}$ are semidirect products and since $\tilde{\phi}_{n} \circ f_{n}^{A}=f_{n}^{B} \circ \phi$, it suffices to check $\tilde{\phi}_{n+1} \circ f_{n+1}^{A}=$ $f_{n+1}^{B} \circ \phi$ as maps from $\operatorname{ker} f_{n}^{A}=A_{H}^{(n)}$ to $H_{1}\left(B ; \mathcal{K} \widetilde{B}_{n}\right)$. For this recall that $f_{n+1}^{A}$ restricted to $\operatorname{ker} f_{n}^{A}$ (and $f_{n+1}^{B}$ restricted to $\phi\left(A_{H}^{(n)}\right) \subset B_{H}^{(n)}=\operatorname{ker} f_{n}^{B}$ ) is given by the canonical map $\left(f_{n}^{A}\right)_{*}$ induced by $\phi$ and $f_{n}^{A}$. On the other hand, $\tilde{\phi}_{n+1}$ restricted to $f_{n+1}^{A}\left(A_{H}^{(n)}\right) \subseteq H_{1}\left(A ; \mathcal{K} \widetilde{A}_{n}\right)$ is determined by $\phi$. Details are left to the reader.

Finally, to establish Property 4, suppose that $\phi: A \rightarrow B$ induces an isomorphism $A / A_{H}^{(m)} \cong B / B_{H}^{(m)}$. Inductively we may assume that this isomorphism extends to isomorphisms $\widetilde{A}_{n} \cong \widetilde{B}_{n}$ and try to show that they extend to an 
isomorphism $\widetilde{A}_{n+1} \cong \widetilde{B}_{n+1}$ as long as $n+1 \leq m$. By the Five Lemma it suffices to show that the modules $H_{1}\left(A ; \mathcal{K} \widetilde{A}_{n}\right)$ and $H_{1}\left(B ; \mathcal{K} \widetilde{B}_{n}\right)$ (modules over isomorphic rings) are isomorphic. But $H_{1}\left(A ; \mathcal{K} \widetilde{A}_{n}\right)$ is completely determined by $H_{1}\left(A ; \mathbb{Z}\left[A / A_{H}^{(n)}\right]\right) /\left\{\mathbb{Z}\left[A / A_{H}^{(n)}\right]-\right.$ Torsion $\}$ together with the inclusion $A / A_{H}^{(n)} \rightarrow \widetilde{A}_{n}$. But the former is precisely $A_{H}^{(n)} / A_{H}^{(n+1)}$. If $n+1 \leq m$ then all of these are carried isomorphically onto the corresponding modules for $B$.

This completes the proof of Theorem 5.6

Corollary 5.11 The torsion-free derived series stabilizes at $n$ if and only if $\left\{\widetilde{G}_{n}\right\}$ stabilizes at $n$. In particular, if $G_{H}^{(n)}=G_{H}^{(n+1)}$, then $\widetilde{G}_{n}=\widetilde{G}_{n+k}$ for all $k \geq 0$ and $\widetilde{G}=\widetilde{G}_{n} \cong\left(\widetilde{G / G_{H}^{(n)}}\right)$. Also, for any group $G,\left(\widetilde{G / G_{H}^{(n)}}\right)_{n}=\left(\widetilde{G / G_{H}^{(n)}}\right)$.

Proof of Corollary 5.11 Suppose that $G_{H}^{(n)}=G_{H}^{(n+1)}$. Then by construction $\widetilde{G}_{n+1}=\widetilde{G}_{n}$. Since $\widetilde{G}_{n}$ stabilizes at $n$, by definition $\widetilde{G}=\widetilde{G}_{n}$. Conversely, if $\widetilde{G}_{n+1}=\widetilde{G}_{n}$ then the kernel of $G \rightarrow \widetilde{G}_{n+1}$ equals the kernel of $G \rightarrow \widetilde{G}_{n}$, thus by Theorem [5.6. $G_{H}^{(n+1)}=G_{H}^{(n)}$. For the final claim, merely note that $G / G_{H}^{(n)}$ is $n$-torsion-free-solvable by Proposition 2.5.

It follows that the torsion-free-solvable completion may be constructed analogously to the Malcev completion.

Corollary $\mathbf{5 . 1 2}$ The torsion-free-solvable completion $\widetilde{G}$ of $G$ is the inverse limit $\left.\lim _{\left(G / G_{H}^{(n)}\right.}\right)$ of the torsion-free-solvable completions of the corresponding quotients by the torsion-free derived series.

Proof of Corollary 5.12 Since $\widetilde{G}$ has been defined as $\lim _{\longleftarrow} \widetilde{G}_{n}$, we must show that $\widetilde{G}_{n}=\left(\widetilde{G / G_{H}^{(n)}}\right)$. By Proposition 2.5 the map $G \rightarrow G / G_{H}^{(n)}$ induces an isomorphism $G / G_{H}^{(n)} \cong\left(G / G_{H}^{(n)}\right) /\left(G / G_{H}^{(n)}\right)_{H}^{(n)}$. Thus, by Theorem[5.6 (4), $\widetilde{G}_{n}=$ $\left(\widetilde{G / G_{H}^{(n)}}\right)_{n}$ which in turn equals $\left(\widetilde{G / G_{H}^{(n)}}\right)$ by the last statement of Corollary 5.11

Example 5.13 If $\beta_{1}(G)=0$, for example if $G$ is a finite group, then the torsion-free-solvable completion and the Malcev completion agree and are both trivial. For we observed in Example 2.6 that the torsion-free derived series stabilizes at $n=0$ in such cases. Thus by Corollary [5.11] $\widetilde{G} \cong \widetilde{G}_{0} \cong 0 \cong$ $M C(G)$. 
Example 5.14 If $G$ is a non-trivial torsion-free abelian group (equivalently $\left.G_{H}^{(1)}=\{e\}\right)$, then $\widetilde{G}$ again agrees with the Malcev completion. For $G_{H}^{(1)}=0$ so the torsion-free derived series stabilizes at $n=1$. Thus by Corollary 5.11 $\widetilde{G} \cong \widetilde{G}_{1}=G \otimes \mathbb{Q} \cong M C(G)$. Note that the algebraic closure of $\mathbb{Z}^{m}$ is $\mathbb{Z}^{m}$ but the Malcev completion and the torsion-free-solvable completion are $\mathbb{Q}^{m}$.

Example 5.15 If $G$ is nilpotent then whereas $G$ /torsion embeds in $\mathrm{MC}(G)$, the torsion-free-solvable completion of $G$ is just the abelianization of $\mathrm{MC}(G)$. For we saw in Example 2.7 that in this case the torsion-free derived series stabilizes at $n \leq 1$. Thus, by Corollary [5.11, $\widetilde{G}=\widetilde{G}_{1}=\left(\widetilde{G / G_{H}^{(1)}}\right)$ and the latter equals $G / G^{(1)} \otimes \mathbb{Q}$ by Example 5.14. This is a flaw in the torsion-free derived series that would be corrected by Remark 5.22

Example 5.16 If $\beta_{1}(G)=1$ then the epimorphism $G \rightarrow \mathbb{Z}$ is rationally 2 connected and so, by Theorem [5.1] it induces isomorphisms $\widetilde{G} \cong \widetilde{\mathbb{Z}} \cong \mathbb{Q} \cong$ $M C(G)$ by Example 5.14 More generally, if $G$ is a group whose classical Alexander module $G_{H}^{(1)} /\left[G_{H}^{(1)}, G_{H}^{(1)}\right]$ has rank zero then, as noted in Example 2.10 the torsion-free derived series stabilizes at $n \leq 1$. Thus, by Corollary 5.11 $\widetilde{G}=\widetilde{G}_{1}=\left(\widetilde{G / G_{H}^{(1)}}\right)=G / G^{(1)} \otimes \mathbb{Q}$.

Example 5.17 If the rank of one of the modules $G_{H}^{(n)} /\left[G_{H}^{(n)}, G_{H}^{(n)}\right]$, is zero then the torsion-free derived series stabilizes at $n$ or less. Thus $\widetilde{G}=\widetilde{G}_{n}$ is solvable by Corollary [5.11] For example if $G$ is the free solvable group $F / F^{(n)}$, then $G$ embeds in its torsion-free-solvable completion since $\left(F / F^{(n)}\right)_{H}^{(\omega)}=0$.

Example 5.18 If $F$ is a non-abelian free group then, since the torsion-free derived series stabilizes at $\omega, \widetilde{F}$ is not nilpotent. In fact, since $F_{H}^{(\omega)}=0, F$ embeds in $\widetilde{F}$. But $\widetilde{F}$ is much larger, for if $G=\pi_{1}\left(S^{3}-L\right)$, where $L$ is a boundary link, then the meridional map $F \rightarrow G$ has a right inverse $G \rightarrow F$ as we saw in Example 2.11 and it follows from Theorem [5.1 that $\widetilde{G} \cong \widetilde{F}$ and $G / G_{H}^{(\omega)}$ embeds in $\widetilde{F}$. Indeed if $G$ is any finitely-presented group with $H_{1}(G ; \mathbb{Q}) \cong \mathbb{Q}^{m}$ and $H_{2}(G ; \mathbb{Q})=0$ then there is a map $F \rightarrow G$ that is $2-$ connected on rational homology and so, by Theorem 5.1] $G / G_{H}^{(\omega)}$ embeds in $\widetilde{F}$. In particular, as we showed in Theorem [5.19] $\widehat{F} /(\widehat{F})_{H}^{(\omega)}$ embeds in $\widetilde{F}$.

\subsection{Relations with the Levine-Vogel algebraic closure}

We now recall the (integral) homological localization functor of Levine-Vogel and discuss the relations between it and the torsion-free-solvable completion. 
J Levine defined the algebraic closure, $\hat{f}: G \rightarrow \widehat{G}$ of a group $G$, unique up to isomorphism [18, page 574]. He pointed out that, if $G$ is finitely presented, it coincides with a notion previously investigated by P. Vogel (see [17]). Specifically, if $X$ is a finite CW-complex with $G=\pi_{1}(X)$ then $\widehat{G}$ is $\pi_{1}(E X)$ where $E X$ is the Vogel localization of $X$. The Levine-Vogel algebraic closure is a universal (integral) homological localization in the following sense. If $A$ is finitely generated, $B$ is finitely presented and $\phi: A \rightarrow B$ is a homomorphism that induces an isomorphism on $H_{1}(-; \mathbb{Z})$, an epimorphism on $H_{2}(-; \mathbb{Z})$, and such that $B$ is the normal closure of $\phi(A)$, then there is an isomorphism $\hat{\phi}: \widehat{A} \rightarrow \widehat{B}$. Moreover the algebraic closure is the "initial" group with this property. Since $\widetilde{G}$ also satisfies this property, we have a canonical map $\widehat{G} \stackrel{\theta}{\rightarrow} \widetilde{G}$ that provides a factorization of $f$ (above) as $G \stackrel{\hat{f}}{\rightarrow} \widehat{G} \stackrel{\theta}{\rightarrow} \widetilde{G}$. This allows us to relate $\widehat{G}$ to $\widetilde{G}$.

Theorem 5.19 Suppose $G$ is a finitely presented group. Then there is a canonical map from the algebraic closure of $G$ to the torsion-free-solvable completion of $G, \widehat{G} \stackrel{\theta}{\rightarrow} \widetilde{G}$ whose kernel is $(\widehat{G})_{H}^{(\omega)}$.

Proof of Theorem [5.19] Recall from [18, Proposition 6] that $\widehat{G}$ can be expressed as $\lim _{i} P_{i}$ where $G=P_{0} \rightarrow P_{1} \rightarrow \cdots \rightarrow P_{k} \rightarrow \cdots \rightarrow \widehat{G}$, each $P_{i}$ is finitely presented and each map satisfies the Levine-Vogel conditions above. Suppose $x \in \operatorname{ker} \theta$. Then $x$ is the image of $p \in P_{k}$ for some $k$, and $p$ lies in the kernel of the induced map $P_{k} \rightarrow \widehat{G} \rightarrow \widetilde{G}$. Since $\widetilde{G} \cong \widetilde{P_{k}}$ by Theorem 5.1 one concludes that $p$ is in the kernel of the canonical map $P_{k} \rightarrow \widetilde{P_{k}}$. Again by Theorem 5.1 $p \in\left(P_{k}\right)_{H}^{(\omega)}$. To now conclude that $x \in(\widehat{G})_{H}^{(\omega)}$, finishing the verification that $\operatorname{ker} \theta \subset(\widehat{G})_{H}^{(\omega)}$, we need the following.

Lemma 5.20 If $B=\lim _{\longrightarrow} P_{i}$ where $P_{i}$ are finitely presented and each map $\phi_{i}: P_{i} \rightarrow P_{i+1}$ induces a monomorphism on $H_{1}(-; \mathbb{Q})$ and an epimorphism on $H_{2}(-; \mathbb{Q})$, then for each $i$ and each $n \leq \omega$, the induced map $\left(P_{i}\right) /\left(P_{i}\right)_{H}^{(n)} \longrightarrow$ $B / B_{H}^{(n)}$ is injective.

Proof of Lemma 5.20 The proof is by induction on $n$. Note that it is trivially true for $n=0$. The proof follows exactly the proof of Theorem 4.1. The Lemma would follow directly if $B$ were finitely related. However this hypothesis on $B$ is only needed in the inductive step to establish the monomorphism conclusion of Proposition 4.3 for the map $H_{1}\left(P_{i} ; \mathcal{K} \Gamma\right) \rightarrow H_{1}(B ; \mathcal{K} \Gamma)$ where $\Gamma=$ $B / B_{H}^{(n)}$. Since each $P_{i}$ is finitely presented, Proposition 4.3 shows that each map $H_{1}\left(P_{i} ; \mathcal{K} \Gamma\right) \rightarrow H_{1}\left(P_{i+k} ; \mathcal{K} \Gamma\right)$ is injective. Thus $H_{1}\left(P_{i} ; \mathcal{K} \Gamma\right) \rightarrow \underset{\lim }{\longrightarrow} H_{1}\left(P_{i+k} ; \mathcal{K} \Gamma\right)$ 
is injective ( $i$ fixed, $k$ varying). Since $B=\lim _{\longrightarrow} P_{i+k}$ and since homology commutes with direct limits [2, page 121], the conclusion of Proposition 4.3 holds for each map $P_{i} \rightarrow B$.

Continuing with the proof of Theorem [5.19, now suppose $x \in(\widehat{G})_{H}^{(\omega)}$. Then $x$ is the image of $p \in P_{i}$ for some $i$ and so, by the case $n=\omega$ of Lemma [5.20 $p \in\left(P_{i}\right)_{H}^{(\omega)}$. Hence, by Theorem [5.1 $p$ is in the kernel of the canonical map $P_{i} \rightarrow \widetilde{\left(P_{i}\right)}$ and since $\widetilde{\left(P_{i}\right)} \cong \widetilde{G}$ we have $x \in \operatorname{ker} \theta$ as desired.

These observations suggest that it might be profitable to consider the functor $G \rightarrow \widehat{G} /(\widehat{G})_{H}^{(\omega)}$ which can be described as a direct $\operatorname{limit} \underset{\longrightarrow}{\lim } P_{i} /\left(P_{i}\right)_{H}^{(\omega)}$. We say that a homomorphism is 2-connected on integral homology or 2-connected if it induces an isomorphism on $H_{1}(-; \mathbb{Z})$ and an epimorphism on $H_{2}(-; \mathbb{Z})$. We say that a homomorphism is 2-connected on rational homology or rationally $2-$ connected if it induces an isomorphism on $H_{1}(-; \mathbb{Q})$ and an epimorphism on $H_{2}(-; \mathbb{Q})$. The interesting question is: If $A \rightarrow B$ is rationally $2-$ connected but fails to be 2-connected with integer coefficients or fails to have the Levine-Vogel normal generation condition, what can be said about the relationship between $\widehat{A}$ and $\widehat{B}$ ? We have the following result, used in [11] to prove that certain invariants of homology cobordism and link concordance are actually invariants of rational homology cobordism.

Corollary 5.21 If $A$ and $B$ are finitely-presented and $\phi: A \rightarrow B$ induces an isomorphism on $H_{1}(-; \mathbb{Q})$ and an epimorphism on $H_{2}(-; \mathbb{Q})$ then $\phi$ induces an embedding $\widehat{A} /(\widehat{A})_{H}^{(\omega)} \subset \widehat{B} /(\widehat{B})_{H}^{(\omega)}$.

Proof of Corollary 5.21 Since any algebraic closure $A \rightarrow \widehat{A}$ is 2-connected with integral coefficients [18. Proposition 4], $\phi$ induces an embedding $\widehat{A} /(\widehat{A})_{H}^{(1)}$ $\subset \widehat{B} /(\widehat{B})_{H}^{(1)}$, since these quotients are merely $H_{1}(-; \mathbb{Z})$ modulo torsion. We proceed by induction. Suppose that $\phi$ induces a monomorphism $\widehat{A} /(\widehat{A})_{H}^{(n-1)} \subset$ $\widehat{B} /(\widehat{B})_{H}^{(n-1)}$. By Proposition [2.3, $\phi$ induces a homomorphism $\widehat{A} /(\widehat{A})_{H}^{(n)} \rightarrow$ $\widehat{B} /(\widehat{B})_{H}^{(n)}$. It suffices to show that this is injective. Suppose $a \in \widehat{A}$ such that $\hat{\phi}(a)=b \in(\widehat{B})_{H}^{(n)}$. As above, we know that $\hat{B}$ can be expressed as $\lim _{\longrightarrow} Q_{i}$ where $B=Q_{0} \rightarrow Q_{1} \rightarrow \cdots \rightarrow Q_{k} \rightarrow \cdots \rightarrow \widehat{B}$, each $Q_{i}$ is finitely presented and each map is 2 -connected with integral coefficients. Similarly, $\widehat{A}=\underline{\lim } P_{i}$. Suppose $q \in Q_{i}$ has image $b$ and $p \in P_{j}$ has image $a$. For simplicity we abbreviate $Q=Q_{i}$ and $P=P_{j}$. By Lemma [5.20] $q \in Q_{H}^{(n)}$. However, since $B \rightarrow Q$ and $A \rightarrow P$ are 2-connected and $A \rightarrow B$ is rationally 2-connected, $\widetilde{B}_{n} \cong \widetilde{Q}_{n}$, $\widetilde{A}_{n} \cong \widetilde{P}_{n}$ and $\widetilde{A}_{n} \cong \widetilde{B}_{n}$ by Theorem [5.6. Also by part 1) of this theorem, the 
image of $q$ under $Q \rightarrow \widetilde{Q}_{n}$ is zero and hence the image of $b$ under $\widehat{B} \rightarrow \widetilde{B} \rightarrow \widetilde{B}_{n}$ is zero. It follows that the image of $a$ under the map $\widehat{A} \rightarrow \widetilde{A}_{n}$ is zero. Consequently the image of $p$ under the map $P \rightarrow \widetilde{P}_{n}$ is zero and hence, by part 1) of Theorem [5.6] $p \in P_{H}^{(n)}$. Thus, by Lemma [5.20, $a \in(\widehat{A})_{H}^{(n)}$. Thus we have shown that $\widehat{A} /(\widehat{A})_{H}^{(n)} \rightarrow \widehat{B} /(\widehat{B})_{H}^{(n)}$ is injective as desired.

Remark 5.22 We are aware that a smaller series (that looks much less natural from an algebraic standpoint) than the torsion-free derived series seems to be more natural from the point of view of rational homology equivalence. Note that $\mathbb{Z}\left[G / G_{H}^{(1)}\right]$ is a Laurent polynomial ring $\mathbb{Z}\left[x_{1}^{ \pm 1}, \ldots, x_{m}^{ \pm 1}\right]$. We could alternatively define $G_{H}^{(2)}$ to be the inverse image of not the full torsion submodule of $G_{H}^{(1)} /\left[G_{H}^{(1)}, G_{H}^{(1)}\right]$, but rather the submodule of elements annihilated by some element of the set $S$ of polynomials whose image under the augmentation map $\mathbb{Z}\left[x_{1}^{ \pm 1}, \ldots, x_{m}^{ \pm 1}\right] \rightarrow \mathbb{Z}$ is non-zero. Then, leaving the definition of the higher $G_{H}^{(n)}$ the same, leads to a series whose terms are smaller than the torsion-free derived series, still containing the derived series, and for which our main theorem remains true because a more precise version of Proposition 4.3 is known to hold (with $\Gamma=\mathbb{Z}^{m}$ and) with the quotient field being replaced by the sub$\operatorname{ring} S^{-1} \mathbb{Z}\left[G / G_{H}^{(1)}\right]$. Generalizing this, a "better" series, $G_{*}^{(n)}$, can be defined as follows. Once $G_{*}^{(n)}$ has been defined, let $C L\left(G / G_{*}^{(n)}\right)$ stand for the Cohn localization of the augmentation $\mathbb{Z}\left[G / G_{*}^{(n)}\right] \rightarrow \mathbb{Q}$. By this we mean the initial ring $\operatorname{map} \mathbb{Z}\left[G / G_{*}^{(n)}\right] \rightarrow C L\left(G / G_{*}^{(n)}\right)$, such that any square matrix over $\mathbb{Z}\left[G / G_{*}^{(n)}\right]$ whose augmentation is invertible over $\mathbb{Q}$ becomes invertible over $C L\left(G / G_{*}^{(n)}\right)$. Note that in the case $n=1, C L\left(G / G_{*}^{(1)}\right)$ is known to be just $S^{-1} \mathbb{Z}\left[G / G_{*}^{(1)}\right]$ with $S$ as above, because since $\mathbb{Z}\left[G / G_{*}^{(1)}\right]$ is commutative, a matrix is invertible if and only if its determinant is invertible. We could then define $G_{*}^{(n+1)}$ to be the kernel of the composition below:

$$
G_{*}^{(n)} \stackrel{\pi_{n}}{\longrightarrow} \frac{G_{*}^{(n)}}{\left[G_{*}^{(n)}, G_{*}^{(n)}\right]}=H_{1}\left(G ; \mathbb{Z}\left[G / G_{*}^{(n)}\right]\right) \longrightarrow H_{1}\left(G ; C L\left(G / G_{*}^{(n)}\right)\right) .
$$

This is in contrast to the torsion-free derived series, wherein $G_{H}^{(n+1)}$ is the kernel of the composition:

$$
G_{H}^{(n)} \stackrel{\pi_{n}}{\longrightarrow} \frac{G_{H}^{(n)}}{\left[G_{H}^{(n)}, G_{H}^{(n)}\right]}=H_{1}\left(G ; \mathbb{Z}\left[G / G_{H}^{(n)}\right]\right) \longrightarrow H_{1}\left(G ; \mathcal{K}\left(G / G_{H}^{(n)}\right)\right) .
$$

The map from $\mathbb{Z}\left[G / G_{*}^{(n)}\right]$ to its quotient field $\mathcal{K}\left(G / G_{*}^{(n)}\right)$ is known to factor through $C L\left(G / G_{*}^{(n)}\right)$. This series is then fully invariant. Much of the structure of our main theorem holds, but there are several problems involving flatness. The Cohn localization is not usually a flat module over $\mathbb{Z}\left[G / G_{*}^{(n)}\right]$ and so we 
have not been able to duplicate the full strength of our main theorem. However we can prove that

$$
G_{r}^{(n)} \subset G_{*}^{(n)} \subset G_{2^{n}}^{r}
$$

and that if $\phi: A \rightarrow B$ is an epimorphism of groups which is rationally 2connected then it induces isomorphisms $A / A_{*}^{(n)} \cong B / B_{*}^{(n)}$ for all $n$. The proofs of these results will appear in a subsequent paper.

\section{References}

[1] K Bousfield, Homological localization towers for groups and $\pi$-modules, Mem. Amer. Math. Soc. 186 (1977) MathReview

[2] KS Brown, Cohomology of groups, Graduate Texts in Mathematics 87, Springer-Verlag, New York (1982) MathReview

[3] A J Casson, Link Cobordism and Milnor's Invariant, Bull. London Math. Soc. 7 (1975) 39-40 MathReview

[4] JC Cha, Injectivity Theorems and Algebraic Closure of Groups with Coefficients Preprint

[5] T D Cochran, Noncommutative knot theory Algebr. Geom. Topol. 4 (2004) 347-398 MathReview

[6] T D Cochran, K E Orr, P Teichner, Knot concordance, Whitney towers and $L^{2}$-signatures, Ann. of Math. (2) 157 (2003) 433-519 MathReview

[7] T D Cochran, K E Orr, P Teichner, Structure in the classical knot concordance group, Comment. Math. Helv. 79 (2004) 105-123 MathReview

[8] TD Cochran, P Teichner, Knot Concordance and von Neumann $\rho-$ invariants, arXiv:math.GT/0411057

[9] P M Cohn, Free rings and their relations, London Mathematical Society Monographs 19, Academic Press Inc. [Harcourt Brace Jovanovich Publishers], London (1985) MathReview

[10] R M Hain, Completions of mapping class groups and the cycle $C-C^{-}$, from: "Mapping class groups and moduli spaces of Riemann surfaces (Göttingen, 1991/Seattle, WA, 1991)", Contemp. Math. 150, Amer. Math. Soc. Providence, RI (1993) 75-105 MathReview

[11] S Harvey, Homology Cobordism Invariants of 3-Manifolds and the CochranOrr-Teichner Filtration of the Link Concordance Group, preprint

[12] S L Harvey, Higher-order polynomial invariants of 3-manifolds giving lower bounds for the Thurston norm, Topology 44 (2005) 895-945 MathReview

[13] P J Hilton, U Stammbach, A course in homological algebra, Graduate Texts in Mathematics 4, Springer-Verlag, New York (1997) MathReview 
[14] J Howie, H R Schneebeli, Homological and topological properties of locally indicable groups, Manuscripta Math. 44 (1983) 71-93 MathReview

[15] T Kim, Filtration of the classical knot concordance group and CassonGordon invariants, Math. Proc. Cambridge Philos. Soc. 137 (2004) 293-306 MathReview

[16] T Kim, An infinite family of non-concordant knots having the same Seifert form, Comment. Math. Helv. 80 (2005) 147-155 MathReview

[17] J LeDimet, Cobordism d'Enlacements de Disques, Bull. Math. Soc. France No.32 116 (1988)

[18] J P Levine, Link concordance and algebraic closure. II, Invent. Math. 96 (1989) 571-592 MathReview

[19] J Lewin, A note on zero divisors in group-rings, Proc. Amer. Math. Soc. 31 (1972) 357-359 MathReview

[20] C Miller, Exponential iterated integrals and the relative solvable completion of the fundamental group of a manifold, Topology 44 (2005) 351-373 MathReview

[21] S Papadima, A I Suciu, Chen Lie algebras, Int. Math. Res. Not. (2004) 10571086 MathReview

[22] D S Passman, The algebraic structure of group rings, Wiley-Interscience [John Wiley \& Sons], New York (1977) MathReview

[23] D Quillen, Rational homotopy theory, Ann. of Math. (2) 90 (1969) 205-295 MathReview

[24] SK Roushon, Topology of 3-manifolds and a Class of Groups II, Boletin de la Sociedad Matematica Mexicana 3a, Serie 10(3) (2004)

[25] J Stallings, Homology and central series of groups, J. Algebra 2 (1965) 170-181 MathReview

[26] B Stenström, Rings of quotients, Springer-Verlag, New York (1975) MathReview

[27] R Strebel, Homological methods applied to the derived series of groups, Comment. Math. Helv. 49 (1974) 302-332 MathReview

[28] P Teichner, Knots, von Neumann signatures, and grope cobordism, from: "Proceedings of the International Congress of Mathematicians, Vol. II (Beijing, 2002)", Higher Ed. Press, Beijing (2002) 437-446 MathReview

[29] G W Whitehead, Elements of homotopy theory, Graduate Texts in Mathematics 61, Springer-Verlag, New York (1978) MathReview 